\title{
Gamma-Ray Blazar Spectra with H.E.S.S. II Mono Analysis: The Case of PKS 2155-304 and PG 1553+113
}

\begin{abstract}
H.E.S.S. Collaboration, H. Abdalla ${ }^{1}$, A. Abramowski ${ }^{2}$, F. Aharonian ${ }^{3,45}$, F. Ait Benkhali ${ }^{3}$, A.G. Akhperjanian ${ }^{\dagger 6,5}$, T. Andersson $^{10}$, E.O. Angüner ${ }^{7}$, M. Arrieta ${ }^{15}$, P. Aubert ${ }^{24}$, M. Backes ${ }^{8}$, A. Balzer ${ }^{9}$, M. Barnard ${ }^{1}$, Y. Becherini ${ }^{10}$, J. Becker Tjus ${ }^{11}$, D. Berge ${ }^{12}$, S. Bernhard $^{13}$, K. Bernlöhr ${ }^{3}$, R. Blackwell ${ }^{14}$, M. Böttcher ${ }^{1}$, C. Boisson ${ }^{15}$, J. Bolmont ${ }^{16}$, P. Bordas ${ }^{3}$, F. Brun ${ }^{26}$, P. Brun ${ }^{17}$, M. Bryan ${ }^{9}$, T. Bulik ${ }^{18}$, M. Capasso $^{29}$, J. Carr ${ }^{19}$, S. Casanova ${ }^{20,3}$, M. Cerruti ${ }^{16}$, N. Chakraborty ${ }^{3}$, R. Chalme-Calvet ${ }^{16}$, R.C.G. Chaves ${ }^{21,22}$, A. Chen ${ }^{23}$, J. Chevalier ${ }^{24}$, M. Chrétien ${ }^{16}$, S. Colafrancesco ${ }^{23}$, G. Cologna ${ }^{25}$, B. Condon ${ }^{26}$, J. Conrad $^{27,28}$, C. Couturier ${ }^{16}$, Y. Cui ${ }^{29}$, I.D. Davids ${ }^{1,8}$, B. Degrange ${ }^{30}$, C. Deil $^{3}$, J. Devin $^{17}$, P. deWilt ${ }^{14}$, L. Dirson ${ }^{2}$, A. Djannati-Ataï ${ }^{31}$, W. Domainko ${ }^{3}$, A. Donath ${ }^{3}$, L.O'C. Drury ${ }^{4}$, G. Dubus ${ }^{32}$, K. Dutson ${ }^{33}$, J. Dyks $^{34}$, T. Edwards $^{3}$, K. Egberts ${ }^{35}$, P. Eger $^{3}$, J.-P. Ernenwein ${ }^{20}$, S. Eschbach ${ }^{36}$, C. Farnier ${ }^{27,10}$, S. Fegan ${ }^{30}$, M.V. Fernandes ${ }^{2}$, A. Fiasson ${ }^{24}$, G. Fontaine ${ }^{30}$, A. Förster ${ }^{3}$, S. Funk ${ }^{36}$, M. Füßling ${ }^{37}$, S. Gabici ${ }^{31}$, M. Gajdus ${ }^{7}$, Y.A. Gallant ${ }^{17}$, T. Garrigoux ${ }^{1}$, G. Giavitto ${ }^{37}$, B. Giebels ${ }^{30}$, J.F. Glicenstein ${ }^{18}$, D. Gottschall ${ }^{29}$, A. Goyal ${ }^{38}$, M.-H. Grondin ${ }^{26}$, D. Hadasch ${ }^{13}$, J. Hahn ${ }^{3}$, M. Haupt ${ }^{37}$, J. Hawkes ${ }^{14}$, G. Heinzelmann ${ }^{2}$, G. Henri' ${ }^{32}$, G. Hermann ${ }^{3}$, O. Hervet ${ }^{15,44}$, A. Hillert ${ }^{3}$, J.A. Hinton ${ }^{3}$, W. Hofmann ${ }^{3}$, C. Hoischen ${ }^{35}$, M. Holler ${ }^{30}$, D. Horns ${ }^{2}$, A. Ivascenko ${ }^{1}$, A. Jacholkowska ${ }^{16}$, M. Jamrozy ${ }^{38}$, M. Janiak ${ }^{34}$, D. Jankowsky ${ }^{36}$, F. Jankowsky ${ }^{25}$, M. Jingo ${ }^{23}$, T. Jogler ${ }^{36}$, L. Jouvin ${ }^{31}$, I. Jung-Richardt ${ }^{36}$, M.A. Kastendieck ${ }^{2}$, K. Katarzyński ${ }^{39}$, U. Katz ${ }^{36}$, D. Kerszberg ${ }^{16}$, B. Khélifi ${ }^{31}$, M. Kieffer ${ }^{16}$, J. King ${ }^{3}$, S. Klepser ${ }^{37}$, D. Klochkov ${ }^{29}$, W. Kluźniak ${ }^{34}$, D. Kolitzus ${ }^{13}$, Nu. Komin ${ }^{23}$, K. Kosack ${ }^{18}$,

S. Krakau ${ }^{11}$, M. Kraus ${ }^{36}$, F. Krayzel ${ }^{24}$, P.P. Krüger ${ }^{1}$, H. Laffon ${ }^{26}$, G. Lamanna ${ }^{24}$, J. Lau ${ }^{14}$, J.-P. Lees ${ }^{24}$, J. Lefaucheur ${ }^{15}$, V. Lefranc ${ }^{18}$, A. Lemière ${ }^{31}$, M. Lemoine-Goumard ${ }^{26}$, J.-P. Lenain*16, E. Leser $^{35}$, T. Lohse ${ }^{7}$, M. Lorentz ${ }^{18}$, R. Liu ${ }^{3}$, R. López-Coto ${ }^{3}$, I. Lypova ${ }^{37}$, V. Marandon ${ }^{3}$, A. Marcowith ${ }^{17}$, C. Mariaud ${ }^{30}$, R. Marx ${ }^{3}$, G. Maurin ${ }^{24}$, N. Maxted ${ }^{14}$, M. Mayer $^{7}$, P.J. Meintjes ${ }^{40}$, M. Meyer ${ }^{27}$, A.M.W. Mitchell ${ }^{3}$, R. Moderski ${ }^{34}$,

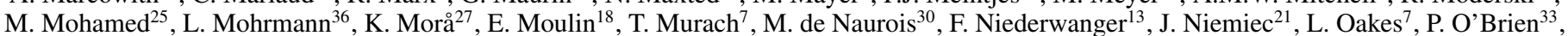
H. Odaka ${ }^{3}$, S. Öttl ${ }^{13}$, S. Ohm ${ }^{37}$, M. Ostrowski ${ }^{38}$, I. Oya ${ }^{37}$, M. Padovani ${ }^{17}$, M. Panter ${ }^{3}$, R.D. Parsons ${ }^{3}$, M. Paz Arribas ${ }^{7}$, N.W. Pekeur ${ }^{1}$,

G. Pelletier ${ }^{32}$, C. Perennes ${ }^{16}$, P.-O. Petrucci ${ }^{32}$, B. Peyaud ${ }^{18}$, S. Pita $^{31}$, H. Poon $^{3}$, D. Prokhorov ${ }^{10}$, H. Prokoph ${ }^{10}$, G. Pühlhofer $^{29}$, M. Punch $^{31,10}$,

A. Quirrenbach ${ }^{25}$, S. Raab ${ }^{36}$, A. Reimer ${ }^{13}$, O. Reimer ${ }^{13}$, M. Renaud ${ }^{17}$, R. de los Reyes ${ }^{3}$, F. Rieger ${ }^{3,41}$, C. Romoli ${ }^{* 4}$, S. Rosier-Lees $^{24}$, G. Rowell $^{14}$,

B. Rudak ${ }^{34}$, C.B. Rulten ${ }^{15}$, V. Sahakian ${ }^{6,5}$, D. Salek ${ }^{42}$, D.A. Sanchez ${ }^{* 24}$, A. Santangelo ${ }^{29}$, M. Sasaki ${ }^{29}$, R. Schlickeiser ${ }^{11}$, F. Schüssler ${ }^{18}$,

A. Schulz ${ }^{37}$, U. Schwanke ${ }^{7}$, S. Schwemmer ${ }^{25}$, M. Settimo ${ }^{16}$, A.S. Seyffert ${ }^{1}$, N. Shafi ${ }^{23}$, I. Shilon ${ }^{36}$, R. Simoni ${ }^{9}$, H. Sol $^{15}$, F. Spanier ${ }^{1}$, G. Spengler ${ }^{27}$, F. Spies ${ }^{2}$, Ł. Stawarz ${ }^{38}$, R. Steenkamp ${ }^{8}$, C. Stegmann ${ }^{35,37}$, F. Stinzing ${ }^{\dagger 3}$, K. Stycz ${ }^{37}$, I. Sushch ${ }^{1}$, J.-P. Tavernet ${ }^{16}$, T. Tavernier ${ }^{31}$, A.M. Taylor ${ }^{* 4}$, R. Terrier ${ }^{31}$, L. Tibaldo ${ }^{3}$, D. Tiziani ${ }^{36}$, M. Tluczykont ${ }^{2}$, C. Trichard ${ }^{20}$, R. Tuffs ${ }^{3}$, Y. Uchiyama ${ }^{43}$, D.J. van der Walt ${ }^{1}$, C. van Eldik $^{36}$, B. van Soelen ${ }^{40}$, G. Vasileiadis ${ }^{17}$, J. Veh $^{36}$, C. Venter ${ }^{1}$, A. Viana ${ }^{3}$, P. Vincent ${ }^{16}$, J. Vink ${ }^{9}$, F. Voisin ${ }^{14}$, H.J. Völk ${ }^{3}$, T. Vuillaume ${ }^{24}$, Z. Wadiasingh ${ }^{1}$, S.J. Wagner ${ }^{25}$, P. Wagner ${ }^{7}$, R.M. Wagner ${ }^{27}$, R. White ${ }^{3}$, A. Wierzcholska ${ }^{21}$, P. Willmann ${ }^{36}$, A. Wörnlein ${ }^{36}$, D. Wouters ${ }^{18}$, R. Yang ${ }^{3}$, V. Zabalza ${ }^{33}$, D. Zaborov ${ }^{* 30}$, M. Zacharias ${ }^{25}$, A.A. Zdziarski ${ }^{34}$, A. Zech ${ }^{15}$, F. Zefi ${ }^{30}$, A. Ziegler ${ }^{36}$, N. Żywucka ${ }^{38}$, and
\end{abstract}

LAT Collaboration, M. Ackermann ${ }^{45}$, M. Ajello ${ }^{46}$, L. Baldini ${ }^{47,48}$, G. Barbiellinii ${ }^{49,40}$, R. Bellazzini ${ }^{51}$, R. D. Blandford ${ }^{48}$, R. Bonino $^{52,53}$, J. Bregeon ${ }^{54}$, P. Bruel ${ }^{30}$, R. Buehler ${ }^{45}$, G. A. Caliandro ${ }^{48,55}$, R. A. Cameron ${ }^{48}$, M. Caragiulo ${ }^{56,57}$, P. A. Caraveo ${ }^{58}$, E. Cavazzuti ${ }^{59}$, C. Cecchi $^{60,61}$, J. Chiang ${ }^{48}$, G. Chiaro ${ }^{62}$, S. Ciprini ${ }^{59,60}$, J. Cohen-Tanugi ${ }^{54}$, F. Costanza ${ }^{57}$, S. Cutini ${ }^{59,60}$, F. D' Ammando ${ }^{63,64}$, F. de Palma ${ }^{47,65}$, R. Desiante ${ }^{66,42}$, N. Di Lalla ${ }^{51}$, M. Di Mauro ${ }^{48}$, L. Di Venere ${ }^{56,57}$, B. Donaggio ${ }^{67}$, C. Favuzzi ${ }^{56,57}$, W. B. Focke ${ }^{48}$, P. Fusco ${ }^{56,57}$, F. Gargano ${ }^{57}$, D. Gasparrini ${ }^{59,60}$, N. Giglietto ${ }^{56,57}$, F. Giordano ${ }^{56,57}$, M. Giroletti ${ }^{63}$, L. Guillemot ${ }^{68,69}$, S. Guiriec ${ }^{70,71}$, D. Horan ${ }^{30}$, G. Jóhannesson ${ }^{72}$, T. Kamae ${ }^{73}$, S. Kensei ${ }^{74}$,

D. Kocevski ${ }^{71}$, S. Larsson ${ }^{75,76}$, J. Li ${ }^{77}$, F. Longo ${ }^{49,50}$, F. Loparco ${ }^{56,57}$, M. N. Lovellette ${ }^{78}$, P. Lubrano ${ }^{60}$, S. Maldera ${ }^{52}$, A. Manfreda ${ }^{51}$, M. N. Mazziotta ${ }^{57}$, P. F. Michelson ${ }^{48}$, T. Mizuno ${ }^{79}$, M. E. Monzani ${ }^{48}$, A. Morselli ${ }^{80}$, M. Negro ${ }^{52,53}$, E. Nuss ${ }^{54}$, M. Orienti ${ }^{63}$, E. Orlando $^{48}$, D. Paneque ${ }^{81}$, J. S. Perkins ${ }^{71}$, M. Pesce-Rollins ${ }^{51,58}$, F. Piron ${ }^{54}$, G. Pivato ${ }^{51}$, T. A. Porter ${ }^{48}$, G. Principe ${ }^{82}$, S. Rainó ${ }^{56,57}$, M. Razzano ${ }^{51,83}$, D. Simone ${ }^{57}$, E. J. Siskind ${ }^{84}$, F. Spada ${ }^{51}$, P. Spinelli ${ }^{56,57}$, J. B. Thayer ${ }^{48}$, D. F. Torres ${ }^{78,85}$, E. Torresi ${ }^{86}$, E. Troja ${ }^{71,87}$, G. Vianello ${ }^{48}$, and K. S. Wood ${ }^{79}$

(Affiliations can be found after the references)

Received XXX; accepted XXX

\section{ABSTRACT}

Context. The addition of a $28 \mathrm{~m}$ Cherenkov telescope (CT5) to the H.E.S.S. array extended the experiment's sensitivity to lower energies. The lowest energy threshold is obtained using monoscopic analysis of data taken with CT5, providing access to gamma-ray energies below $100 \mathrm{GeV}$ for small zenith angle observations. Such an extension of the instrument's energy range is particularly beneficial for studies of Active Galactic Nuclei (AGNs) with soft spectra, as expected for those at a redshift $\geq 0.5$. The high-frequency peaked BL Lac objects PKS 2155-304 $(z=0.116)$ and PG $1553+113(0.43<z<0.58)$ are among the brightest objects in the gamma-ray sky, both showing clear signatures of gamma-ray absorption at $E>100 \mathrm{GeV}$ interpreted as being due to interactions with the extragalactic background light (EBL).

Aims. This work is directed toward a twofold aim: to demonstrate the monoscopic analysis of CT5 data with a low energy threshold, and to obtain accurate measurements of the spectral energy distributions (SED) of PKS 2155-304 and PG 1553+113 near their SED peaks at energies $\approx$ $100 \mathrm{GeV}$.

Methods. Multiple observational campaigns of PKS 2155-304 and PG 1553+113 were conducted during 2013 and 2014 using the full H.E.S.S. II instrument (CT1-5). A monoscopic analysis of the data taken with the new CT5 telescope was developed along with an investigation into the systematic uncertainties on the spectral parameters which are derived from this analysis.

Results. Using the data from CT5, the energy spectra of PKS 2155-304 and PG 1553+113 were reconstructed down to conservative threshold energies of $80 \mathrm{GeV}$ for PKS 2155-304, which transits near zenith, and $110 \mathrm{GeV}$ for the more northern PG 1553+113. The measured spectra, well fitted in both cases by a log-parabola spectral model (with a $5.0 \sigma$ statistical preference for non-zero curvature for PKS $2155-304$ and $4.5 \sigma$ for PG 1553+113), were found consistent with spectra derived from contemporaneous Fermi-LAT data, indicating a sharp break in the observed spectra of both sources at $E \approx 100 \mathrm{GeV}$. When corrected for EBL absorption, the intrinsic H.E.S.S. II mono and Fermi-LAT spectrum of PKS 2155-304 was found to show significant curvature. For PG 1553+113, however, no significant detection of curvature in the intrinsic spectrum could be found within statistical and systematic uncertainties.

Key words. galaxies: active - BL Lacertae objects: individual: PKS 2155-304, PG 1553+113 - gamma rays: galaxies 


\section{Introduction}

The very high energy (VHE, $E \gtrsim 100 \mathrm{GeV}$ ) gamma-ray experiment of the High Energy Stereoscopic System (H.E.S.S.) consists of five imaging atmospheric Cherenkov telescopes (IACTs) located in the Khomas Highland of Namibia $\left(23^{\circ} 16^{\prime} 18^{\prime \prime} \mathrm{S}\right.$, $\left.16^{\circ} 30^{\prime} 00^{\prime \prime} \mathrm{E}\right), 1835 \mathrm{~m}$ above sea level. From January 2004 to October 2012, the array was operated as a four telescope instrument (H.E.S.S. phase I). The telescopes, CT1-4, are arranged in a square formation with a side length of $120 \mathrm{~m}$. Each of these telescopes has an effective mirror surface area of $107 \mathrm{~m}^{2}$, a field of view of $5^{\circ}$ in diameter, capable of detecting cosmic gamma rays in the energy range $0.1-100 \mathrm{TeV}$ (Aharonian et al. 2006a) In October 2012 a fifth telescope, CT5, placed at the centre of the original square, started taking data. This set-up is referred to as H.E.S.S. phase II, or H.E.S.S. II. With its effective mirror surface close to $600 \mathrm{~m}^{2}$ and a fast, finely pixelated camera (Bolmont et al. 2014), CT5 potentially extends the energy range covered by the array down to energies of $\sim 30 \mathrm{GeV}$.

In this study, we focus on obtaining high statistic results with observations of the high-frequency peaked BL Lac objects PKS 2155-304 and PG 1553+113. These blazars are among the brightest objects in the VHE gamma-ray sky. Furthermore, the spectra of both these blazars exhibit signatures of gamma-ray absorption at energies $E \sim 100 \mathrm{GeV}$, due to interactions with the extragalactic background light (EBL).

PKS 2155-304 is a high-frequency peaked BL Lac (HBL) object at $z=0.116$ (Ganguly et al. 2013). This source is located in a galaxy poor cluster (Falomo et al. 1993) and the host galaxy is resolved (Kotilainen et al. 1998). It was first discovered as a high energy emitter by the HEAO 1 X-ray satellite (Griffiths et al. 1979; Schwartz et al. 1979) Gamma-ray emission in the energy range $30 \mathrm{MeV}$ to $10 \mathrm{GeV}$ was detected from this blazar by the EGRET instrument on board the Compton Gamma Ray Observatory (Vestrand et al. 1995). The first detection in the VHE range was attained in 1996 by the University of Durham Mark 6 Telescope, with a statistical significance of $6.8 \sigma$ (Chadwick et al. 1999). Starting from 2002 the source was regularly observed with H.E.S.S., with the first detection based on the 2002 data subsequently published with just one telescope of H.E.S.S. phase I (Aharonian et al. 2005). After completion of the array, this source was detected in stereoscopic mode in 2003 with high significance $(>100 \sigma)$ at energies greater than 160 $\mathrm{GeV}$ (Aharonian et al. 2005). Strong flux variability with multiple episodes of extreme flaring activity in the VHE band were reported (Aharonian et al. 2007; H.E.S.S. Collaboration et al. 2010; Aleksić et al. 2012a) A photon index 1 of $3.53 \pm 0.06_{\text {stat }} \pm$ $0.10_{\text {syst }}$ was obtained from analysis of observations during a low flux state (2005-2007) above $200 \mathrm{GeV}$ (H.E.S.S. Collaboration et al. 2010). For average and high flux states the presence of curvature or a cut-off was favoured from the spectral fit analysis carried out (H.E.S.S. Collaboration et al. 2010)

The HBL object PG 1553+113 was first announced as a VHE gamma-ray source by H.E.S.S. (Aharonian et al. 2006b) and independently and almost simultaneously confirmed by MAGIC using observations from 2005 (Albert et al. 2007) The H.E.S.S. I measurements (Aharonian et al. 2008) yielded a photon index $\Gamma=4.5 \pm 0.3_{\text {stat }} \pm 0.1_{\text {syst }}$ above $225 \mathrm{GeV}$. At high energies

Send offprint requests to: H.E.S.S. and LAT collaborations, e-mail: contact.hess@hess-experiment.eu.

* Corresponding Authors

$\dagger$ Deceased

1 Where the photon index, $\Gamma$, describes the spectral shape of the photon energy distribution, $d N / d E \propto E^{-\Gamma}$.
(HE, $100 \mathrm{MeV}<E<300 \mathrm{GeV}$ ) the source was detected by Fermi-LAT with a photon index of $1.68 \pm 0.03$ (Abdo et al. 2009, 2010), making PG $1553+113$ an active galactic nucleus (AGN) with one of the largest HE-VHE spectral breaks observed and a hint for long-term gamma-ray flux oscillation (Ackermann et al. 2015). The redshift of PG $1553+113$ is constrained by UV observations to the range $0.43<z \lesssim 0.58$ (Danforth et al. 2010). The first upper-limits of $z<0.69$ (pre-Fermi-LAT) Mazin \& Goebel (2007) and more recently (post-Fermi-LAT) $z<0.61$ on the source redshift have been obtained Aliu et al. (2015) using $\mathrm{TeV}$ data and of $z<0.53$ by Biteau \& Williams (2015) using also $\mathrm{GeV}$ data. Assuming that the difference in spectral indices between the HE and VHE regimes is imprinted by the attenuation by the extragalactic background light, the redshift was constrained to the range $z=0.49 \pm 0.04$ (Abramowski et al. 2015).

This paper reports on the first observations of PKS 2155-304 and PG 1553+113 conducted in 2013 and 2014 using the H.E.S.S. II instrument (CT5) in monoscopic mode. A description of the analysis for both AGNs, using data from this instrument, is provided. Systematic errors associated with our results are also estimated. Particular emphasis is placed on the spectral measurements at low energies and their connection with the Fermi-LAT measurements. Using the H.E.S.S. II mono and Fermi-LAT results, the implications on intrinsic source spectrum are considered.

\section{The H.E.S.S. II experiment}

The H.E.S.S. II experiment is the first hybrid Cherenkov instrument and has the ability to take data in different modes. The H.E.S.S. II system triggers on events detected either by CT5 only (mono) or by any combination of two or more telescopes (stereo, CT5 plus at least one of CT1-4, or at least two of CT1-4). The field of view of CT5 is $3.2^{\circ}$ in diameter, smaller than that for CT1-4. Consequently, not all stereo triggers include CT5. The standard observation mode of H.E.S.S. II is to collect both mono and stereo events during the same observation run.

The analysis of CT1-5 stereo data provides a lower energy threshold, better hadron rejection and better angular resolution than with CT1-4 only. The analysis of H.E.S.S. II mono events potentially provides a factor of $\approx 4$ lower energy threshold than CT1-5 stereo. However, the absence of stereoscopic constraints makes the rejection of hadronic events more difficult, leading to a larger background and reduced signal-to-background ratio at the analysis level. The low energy threshold of H.E.S.S. II mono implies high event rates, and thus small statistical uncertainties on the background, which leads to tight requirements for the accuracy of background subtraction. The angular reconstruction of the monoscopic analysis is significantly less precise than that obtained in the stereoscopic mode, leading to a reduction of the sensitivity for point-like sources.

Nevertheless, the H.E.S.S. II mono analysis provides new opportunities to probe astronomy at energies $<100 \mathrm{GeV}$ for southern sources, which are complementary to satellite experiments (e.g. Fermi Large Area Telescope, LAT) and to northern hemisphere facilities such as MAGIC and VERITAS which can detect northern sources below $100 \mathrm{GeV}$ (Aleksić et al. 2015a; Abeysekara et al. 2015). The low energy threshold provided by H.E.S.S. II mono is, consequently, particularly beneficial for studies of bright variable objects such as gamma-ray bursts and active galactic nuclei (AGNs) out to high redshifts $(z \gtrsim 0.5)$, along with associated spectral features introduced into the spectra through gamma-ray interactions with the extragalactic background light (EBL). 
The full performance characterization of the CT1-5 system will be provided in a forthcoming publication.

\section{H.E.S.S. II Mono Observations and Analysis}

\subsection{H.E.S.S. II Observations}

PKS 2155-304 was monitored with H.E.S.S. II regularly for two consecutive years: in 2013 (from Apr 21 to Nov 5, 2013, MJD 56403-56601); and 2014 (May 28 - Jun 9, 2014, MJD 5680556817). PG 1553+113 was observed with H.E.S.S. II between May 29 and Aug 9, 2013 (MJD 56441-56513). Most of the observations were taken using the full H.E.S.S. II array. This paper only reports on the monoscopic analysis of this data, which provides the lowest achievable energy threshold.

H.E.S.S. data taking is organised in 28 min blocks, called runs. Observations are usually taken in wobble mode, with the camera's field of view centred at a $0.5^{\circ}$ or $0.7^{\circ}$ offset from the source position, in either direction along the right-ascension or declination axis. Only runs for which the source position is located between $0.35^{\circ}$ and $1.2^{\circ}$ off-axis from camera centre are used in the present analysis ${ }^{2}$. This is to ensure that the source is well within the field of view and allow background subtraction using the reflected-region background method (Berge et al. 2007).

\subsection{Data Quality Selection}

To ensure the quality of the AGN data sets for the H.E.S.S. II mono analysis the following run quality criteria were applied.

- Stable clear sky conditions according to the telescope radiometers 3 .

- Relative humidity $<90 \%$;

- Run duration ${ }^{4}>5 \mathrm{~min}$ and live time fraction $>90 \%$;

- At least $90 \%$ of pixels in CT5 are active (pixels can be temporarily switched off due to a star in the field of view or removed from the data due to bad calibration);

- CT5 trigger in standard configuration pixel/sector threshold $=4 / 2.5$, see Aharonian et al. (2006a) for a definition of the trigger pattern;

- CT5 trigger rate between 1200 and $3000 \mathrm{~Hz}$ (its nominal value depends on the observed field of view and zenith angle) and stable within $\pm 10 \%$ during a run;

- Telescope tracking functioning normally;

\subsection{Data Analysis}

The data sets were processed with the standard H.E.S.S. analysis software using the Model reconstruction (de Naurois \& Rolland 2009) which was recently adapted to work with monoscopic events (Holler et al. 2015) The Model reconstruction performs a likelihood fit of the air shower image to a semi-analytical model of an average gamma-ray shower parameterised as a function of energy, primary interaction depth, impact distance and direction. Gamma-like candidate events are selected based on the value of

\footnotetext{
2 Runs with non-standard wobble offests were taken during the commissioning phase to assess the performance of the instrument.

3 We use the narrow field-of-view radiometers installed on the CT14 telescopes, requesting radiometer temperature to be less than $-20^{\circ} \mathrm{C}$ and stable during the run within $\pm 3{ }^{\circ} \mathrm{C}$.

4 A run may be interrupted due to an automated target-of-opportunity observation of a transient source, deteriorating weather conditions, or a technical issue.
}

the goodness-of-fit variable and the reconstructed primary interaction depth. In addition, events with an estimated error in direction reconstruction $>0.3^{\circ}$ are rejected. The low energy threshold is controlled with a dedicated variable NSB Goodness, which characterises the likelihood of accidentally triggering on fluctuations due to the night sky background. Two cut configurations were defined for this analysis, loose and standard, with different settings for the NSB Goodness cut. Loose cuts provide the lowest energy threshold, but may lead to a significant level of systematic errors in the background subtraction when applied to high statistics datasets. Standard cuts provide a better control over the background subtraction at the cost of increased threshold. The event selection cuts, except for the NSB Goodness cut, were optimised to maximise the discovery potential for a point source with a photon index of 3.0 observed at a zenith angle of $18^{\circ}$ for $5 \mathrm{hr}$. The optimized analysis provides an angular resolution of $\approx$ $0.15^{\circ}$ (68\% containment radius) at $100 \mathrm{GeV}$ and energy resolution of $\approx 25 \%$. For photon indices harder than 3.0, standard cuts provide a better sensitivity than loose cuts.

The background subtraction is performed using the standard algorithms used in H.E.S.S.- the ring background method (for sky maps) and the reflected-region background method (with multiple off-source regions, for spectral measurements Berge et al. 2007). The ring background method uses a zenithdependent two-dimensional acceptance model, an inner ring radius of $0.3^{\circ}$ and outer radius of $0.6^{\circ}$, and top-hat smoothing radius of $0.1^{\circ}$. The acceptance model, which describes the observed distribution of background events in the camera's field of view in absence of gamma-ray sources, is obtained from the data itself, using background events outside of a radius of $0.3^{\circ}$ from any known VHE gamma-ray source (for this analysis, PKS 2155-304 and PG 1553+113). The reflected-region background method uses an on-source region radius of $0.122^{\circ}$, which corresponds to an angular distance cut $\theta^{2}<0.015 \mathrm{deg}^{2}$. The number of off-source regions was adjusted on a run-by-run basis so as to always use the maximum possible number of them, given the wobble angle. For instance, for a wobble angle of $0.5^{\circ}$ nine off-source regions were used. A simple acceptance model, which only corrects for linear gradients in the acceptance, is used with this method. The significance of the excess after background subtraction is determined using the method described by $\mathrm{Li} \&$ Ma (1983). Spectral measurements are obtained using the forward folding technique (Piron et al. 2001), applied to the excess events observed with the reflected-region background method. The energy threshold for the spectral fit is defined as the energy at which the effective area reaches $15 \%$ of its maximum value, in line with the definition previously adopted in H.E.S.S. analysis (H.E.S.S. Collaboration et al. 2014a). Such a definition ensures that the systematic uncertainties in the analysis are kept under control. The H.E.S.S. II mono analysis was applied to all events that include CT5 data (ignoring information from CT1-4).

\section{Results}

\subsection{PKS 2155-304}

The PKS 2155-304 data set, filtered as explained in Sect. 3.2. comprises 138 runs. The total live time of this data set is $56.0 \mathrm{hr}$, 43.7 hr taken in 2013 and 12.3 hr taken in 2014. During these observations, the source zenith angle ranged from $7^{\circ}$ to $60^{\circ}$, with a median value of $16^{\circ}$. This data set was analysed using standard cuts as described in Sect. 3.3 The background event counts obtained for the off-source regions in each run (in the reflectedregion background analysis) were used to perform an additional 


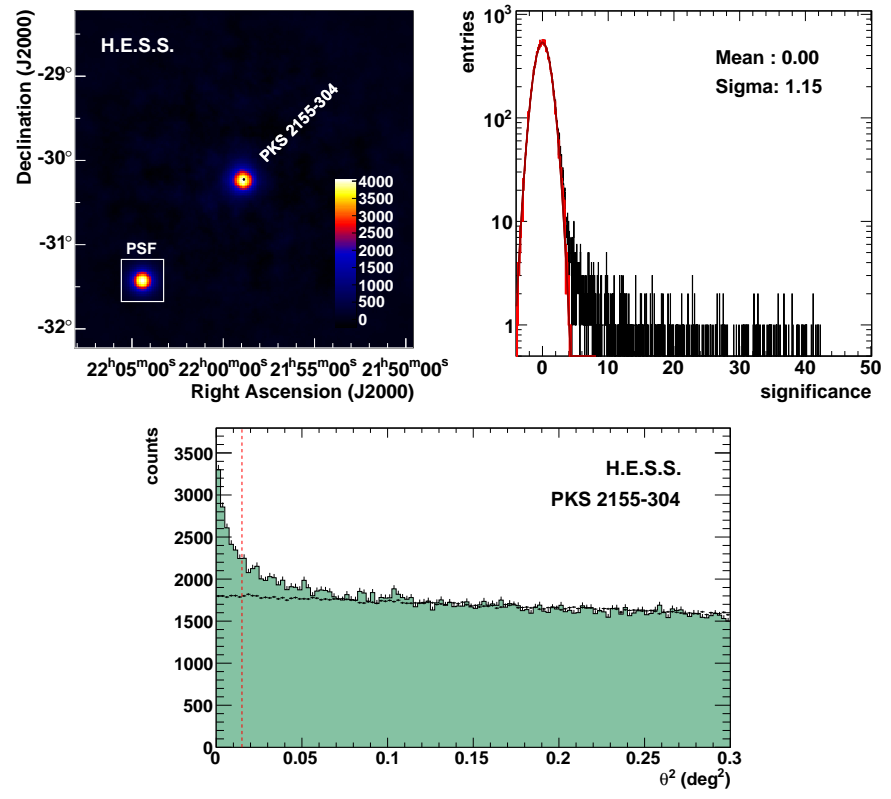

Fig. 1. Top: (Left) Excess map of events observed in the direction of PKS 2155-304 using the H.E.S.S. II mono analysis (2013-2014 data). The inset represents the point spread function of the instrument obtained from simulations. The source position is indicated by a black dot. (Right) The significance distribution that corresponds to the excess map (black histogram). The distribution obtained by excluding a circular region of $0.3^{\circ}$ radius around the source is shown in red; the results of a Gaussian fit to this distribution are also shown. Bottom: The distribution of $\theta^{2}$ (squared angular distance to PKS 2155-304) for gamma-like events obtained with the H.E.S.S. II mono analysis (filled histogram) in comparison with the normalised $\theta^{2}$ distribution for off-source regions (black points). The vertical dashed line shows the limit of the on-source region. The energy threshold for this analysis is $\approx 80 \mathrm{GeV}$

test of the uniformity of the camera acceptance. This was done using a likelihood ratio test (LLRT), with the baseline hypothesis that the event counts observed in all off-source regions come from the same Poisson distribution, and a nested model allowing for different mean values in each region. The results of this test were consistent with an axially-symmetric camera acceptance.

The sky map obtained for PKS 2155-304 using the H.E.S.S. II mono analysis is shown in the top-left panel of Fig. 1 The analysis found that the source is detected with a significance of $\approx 42 \sigma$, with $\approx 4000$ excess events. The corresponding distribution of the excess significance of all skymap bins is shown in the top-right panel of Fig. 1. The width of the observed excess is approximately compatible with the simulated point spread function (PSF; shown in the inset on Fig. 11). The best-fit position of the excess is found $32^{\prime \prime} \pm 10^{\prime \prime}$ stat from the target position. This offset can be attributed to the systematic errors on the telescope pointing. Outside the exclusion radius of $0.3^{\circ}$ the significance distribution was found to be well fit by a Gaussian with $\sigma=1.149 \pm 0.004$. This result indicates the presence of a systematic effect in background subtraction, whose $\sigma_{\text {syst }}$ corresponds to about $57 \%$ of the statistical error ${ }^{5}\left(\sigma_{\text {stat }}=1.0\right.$ by construction $)$.

\footnotetext{
5 We here assume that the errors add in quadrature. A value of $\sigma=$ $\sqrt{1+\sigma_{\text {syst }}^{2}}>\sqrt{2}$ would then indicate the dominance of background subtraction errors.
}

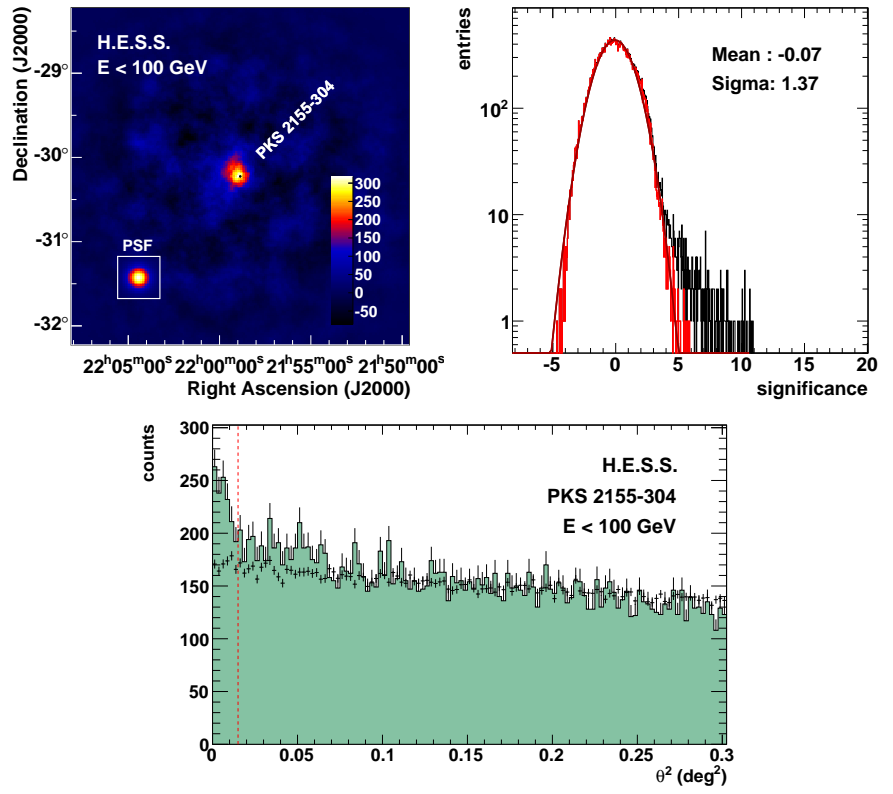

Fig. 2. Top : The PKS 2155-304 excess map (left) and significance distribution (right) for events with reconstructed energy $E<100 \mathrm{GeV}$ (H.E.S.S. II mono analysis, 2013-2014 data) Bottom: The distribution of $\theta^{2}$ (squared angular distance to PKS 2155-304) for gamma-like events.

This effectively reduces the observed excess significance from $42 \sigma$ to $\approx 36 \sigma^{6}$. This systematic effect is currently under investigation as part of a larger effort to understand the mono analysis performance. Repeating the analysis using only events with reconstructed energy below $100 \mathrm{GeV}$ leads to a $10 \sigma(7.3 \sigma)$ significance at the position of PKS 2155-304 in the skymap (Fig. 2). The significance distribution outside the exclusion region has $\sigma=1.374 \pm 0.005$, indicating that the background subtraction errors are slightly smaller than the statistical errors. Thus the source is confidently detected at $E<100 \mathrm{GeV}$.

The distribution of $\theta^{2}$, the square of the angular difference between the reconstructed shower position and the source position, is shown in the bottom panel of Fig. 11 (filled histogram). A $43 \sigma$ excess over the background (black crosses) is observed within the on-source region $\left(\theta^{2}<0.015 \mathrm{deg}^{2}\right)$.

The reconstructed spectrum of PKS 2155-304 obtained for 2013, and each of the observation years (2013 and 2014), is shown in Figs. 3 and 4, respectively. For the full data set $(2013+2014)$, a log-parabola model, $\mathrm{d} N / \mathrm{d} E=$ $\Phi_{0}\left(E / E_{0}\right)^{-\Gamma-\beta \cdot \log \left(E / E_{0}\right)}$, better fits the data with respect to a simple power-law model with a log-likelihood ratio of 25 (i.e. $5 \sigma$ ). The flux normalisation is found to be $\Phi_{0}=\left(5.11 \pm 0.15_{\text {stat }}\right) \times$ $10^{-10} \mathrm{~cm}^{-2} \mathrm{~s}^{-1} \mathrm{TeV}^{-1}$ at a decorrelation ${ }^{7}$ energy $E_{0}=156 \mathrm{GeV}$, with a photon index $\Gamma=2.63 \pm 0.07_{\text {stat }}$ and a curvature parameter $\beta=0.24 \pm 0.06_{\text {stat }}$. The spectral data points (blue filled circles) cover the energy range from $80 \mathrm{GeV}$ to $1.2 \mathrm{TeV}$ (not including upper limits). The spectral parameters obtained for the 2013 and 2014 data sets are given in Table 1 . The isotropic luminosity that

${ }^{6}$ From this point forward, significance values are not corrected for this effect, with the corrected values being quoted within brackets immediately proceeding these uncorrected values.

${ }^{7}$ For the log-parabola model, the decorrelation energy is the energy where the error on the flux is the smallest, i.e. where the confidence band butterfly is the narrowest in the graphical representation. 
Table 1. Spectral analysis results of H.E.S.S. II mono observations. For both blazars, the observational period is provided along with the spectral parameters: decorrelation energy $E_{0}$; differential flux at the decorrelation energy $\Phi_{0}$; photon index $\Gamma$; and curvature parameter $\beta$. These three parameters describe the log-parabola fit to the spectra.

\begin{tabular}{cccccccc}
\hline \hline Source & Year & MJD & $\begin{array}{c}\text { Livetime } \\
{[\mathrm{hr}]}\end{array}$ & $\begin{array}{c}E_{0} \\
{[\mathrm{GeV}]}\end{array}$ & $\begin{array}{c}\Phi_{0} \\
{\left[10^{-9} \mathrm{~cm}^{-2} \mathrm{~s}^{-1} \mathrm{TeV}^{-1}\right]}\end{array}$ & $\Gamma$ & $\beta$ \\
\hline PKS 2155-304 & 2013 & $56403-56601$ & 43.7 & 151 & $0.530 \pm 0.018_{\text {stat }}$ & $2.65 \pm 0.09_{\text {stat }}$ & $0.22 \pm 0.07_{\text {stat }}$ \\
& 2014 & $56805-56817$ & 12.3 & 177 & $0.532 \pm 0.029_{\text {stat }}$ & $2.82 \pm 0.13_{\text {stat }}$ & $0.16 \pm 0.10_{\text {stat }}$ \\
& $2013+2014$ & $56403-56817$ & 56.0 & 156 & $0.511 \pm 0.015_{\text {stat }}$ & $2.63 \pm 0.07_{\text {stat }}$ & $0.24 \pm 0.06_{\text {stat }}$ \\
\hline PG 1553+113 & 2013 & $56441-56513$ & 16.8 & 141 & $1.48 \pm 0.07_{\text {stat }}$ & $2.95 \pm 0.23_{\text {stat }}$ & $1.04 \pm 0.31_{\text {stat }}$ \\
\hline \hline
\end{tabular}

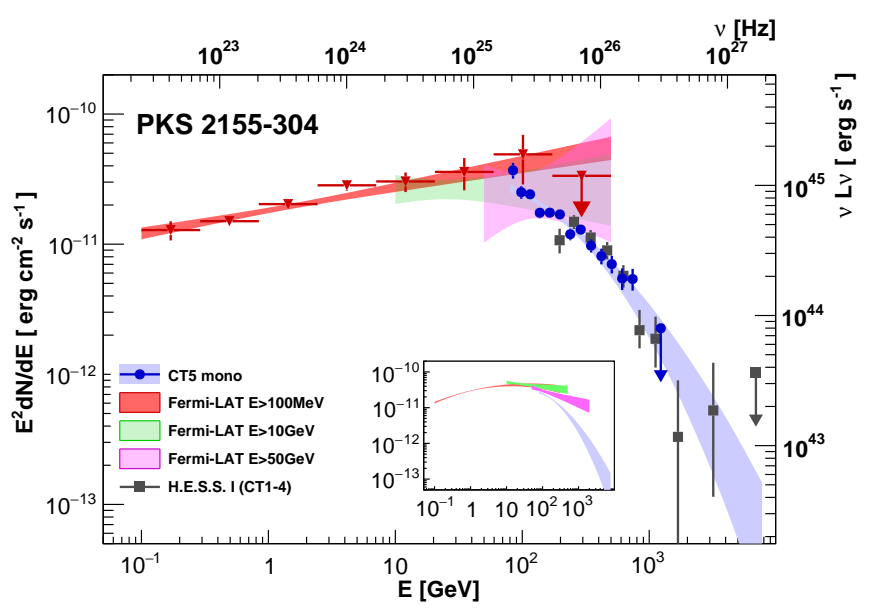

Fig. 3. The energy spectrum of PKS 2155-304 obtained from the H.E.S.S. II mono analysis (2013 data, shown by blue circles with confidence band) in comparison with the contemporaneous Fermi-LAT data with an energy threshold of $0.1 \mathrm{GeV}$ (red triangles and confidence band), $10 \mathrm{GeV}$ (green band), and $50 \mathrm{GeV}$ (purple band) and contemporaneous CT1-4 data (grey squares). In all cases the confidence bands represent the $1 \sigma$ region. The right-hand $y$-axis shows the equivalent isotropic luminosity (not corrected for beaming or EBL absorption). The inset compares the H.E.S.S. confidence band with the Fermi-LAT catalogue data (3FGL, 1FHL and 2FHL, see Sect.4.4.2).

corresponds to the measured SED is shown by the additional yaxis on the right-hand side of the SED plots.

\section{2. $P G 1553+113$}

The PG $1553+113$ data set, filtered as explained in Sect. 3.2 comprises 39 runs (16.8 hr live time), which were analysed using loose cuts as described in Sect. 3.3. This analysis configuration, providing lower energy threshold than standard cuts, is well suited for bright soft-spectrum sources, such as PG 1553+113. During the observations, the source zenith angle ranged between $33^{\circ}$ and $40^{\circ}$, with a mean value of $35^{\circ}$. The sky map obtained for PG $1553+113$ using the H.E.S.S. II mono analysis is shown in the top-left panel of Fig. 5. This analysis found that the source is detected with a statistical significance of $27 \sigma(21 \sigma)$, with $\approx$ 2500 excess events.

The best-fit position of the excess is found to be $36^{\prime \prime} \pm 12^{\prime \prime}{ }_{\text {stat }}$ from the target position, this shift is attributed to the systematic errors on the telescope pointing. The width of the observed excess is compatible with the simulated PSF within a $10 \%$ systematic uncertainty on the PSF width.

The significance distribution in the region outside of the $0.3^{\circ}$ exclusion radius is consistent with a normal distribution (top-

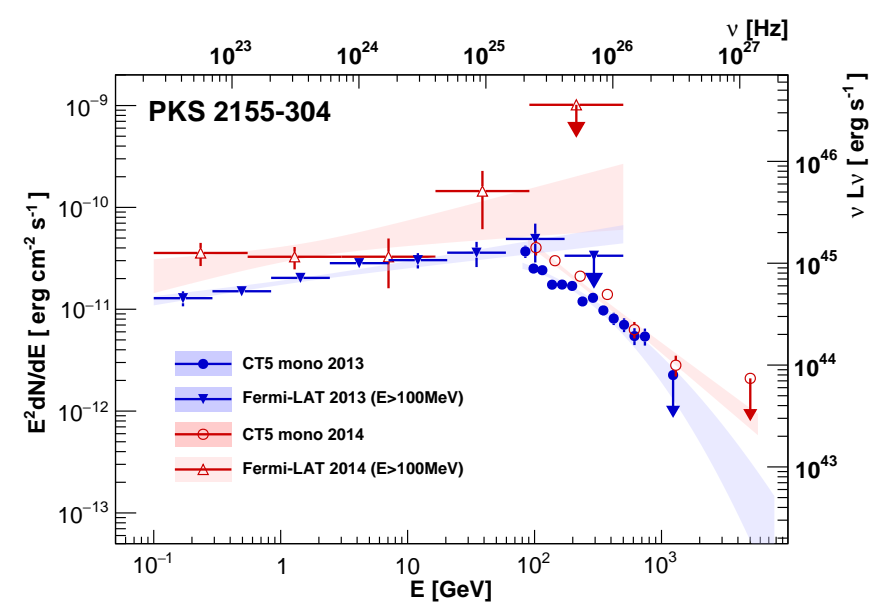

Fig. 4. The SED of PKS 2155-304 separated into the 2013 and 2014 observation periods. Both the H.E.S.S. II mono and contemporaneous Fermi-LAT data are shown. The bands represent the $1 \sigma$ confidence region.

right panel of Fig. 57. The same holds true when the analysis is repeated in only a low energy bin, with a reconstructed energy range of $100-136 \mathrm{GeV}$. Within this energy bin, the source is detected with a $10 \sigma(8.2 \sigma)$ significance (Fig. 6). The significance distribution outside the exclusion region has $\sigma=1.219 \pm 0.005$ and $1.288 \pm 0.005$, for the full energy range and the first energy bin, respectively, indicating presence of background subtraction errors at a level smaller than the statistical errors.

The $\theta^{2}$ distribution is shown in the bottom panel of Fig. 5. A $27 \sigma(21 \sigma)$ excess over the background is observed within the on-source region $\left(\theta^{2}<0.015 \mathrm{deg}^{2}\right)$. The reconstructed spectrum, with a threshold of $110 \mathrm{GeV}$, is found to be well fit by a logparabola (with a LLRT of 20 over the power-law model, Fig. 7), with a photon index $\Gamma=2.95 \pm 0.23_{\text {stat }}$ at decorrelation energy $E_{0}=141 \mathrm{GeV}$, curvature parameter $\beta=1.04 \pm 0.31_{\text {stat }}$, and differential flux $\Phi_{0}=\left(1.48 \pm 0.07_{\text {stat }}\right) \times 10^{-9} \mathrm{~cm}^{-2} \mathrm{~s}^{-1} \mathrm{TeV}^{-1}$ at $E_{0}$. The spectral data points (blue filled circles) cover the energy range from $110 \mathrm{GeV}$ to $550 \mathrm{GeV}$ (not including upper limits).

\subsection{Cross check analysis}

The robustness of the new H.E.S.S. II mono results presented above has been tested through an independent analysis using the Image Pixel-wise fit for Atmospheric Cherenkov Telescopes (ImPACT) method described in Parsons \& Hinton (2014). This independent analysis provides a consistent cross-check with the above results, being successfully applied to the reconstruction of data coming from CT5-only triggers (Parsons et al. 2015). The analysis was equally capable of detecting PKS 2155-304 below $100 \mathrm{GeV}$ and the derived spectra were found to be in very good 


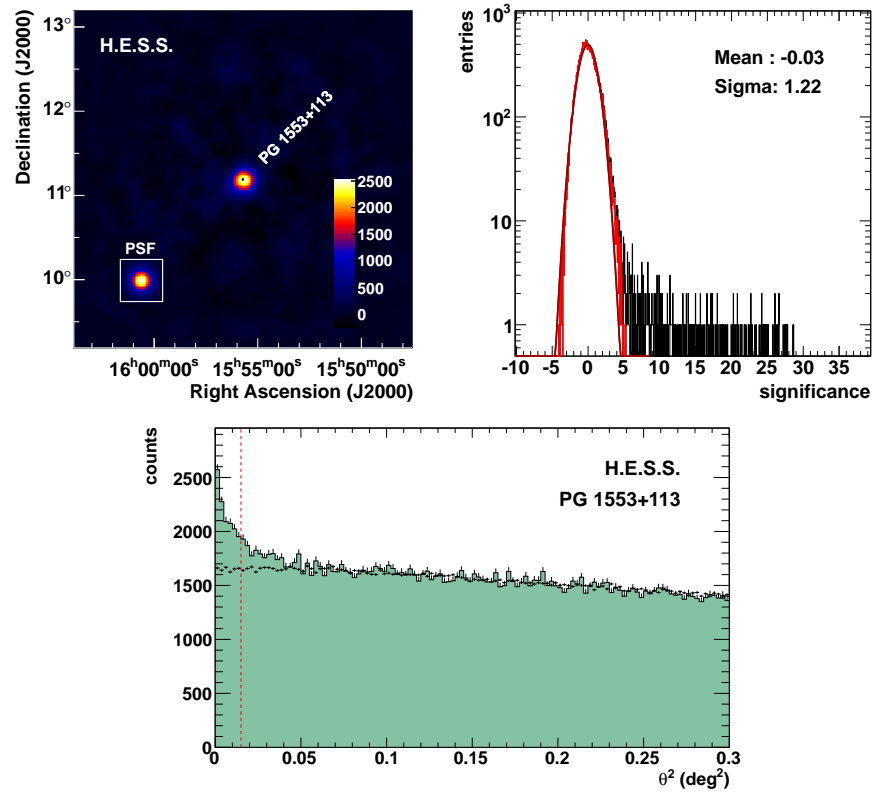

Fig. 5. Top: (Left) Excess map of events observed in the direction of PG $1553+113$ using the H.E.S.S. II mono analysis (16.8 hr live time). The source position is indicated by a black dot. (Right) The significance distribution that corresponds to the excess map. The meaning of the histograms and statistics data is the same as in Fig. 1] Bottom: The $\theta^{2}$ distribution for PG $1553+113$. The meaning of the data shown is the same as in Fig. 1. The vertical dashed line shows the limit of the on-source region. The energy threshold for this analysis is $\approx 100 \mathrm{GeV}$.

agreement with the Model analysis for both PKS 2155-304 and PG 1553+113. Furthermore, the difference between the spectral parameters derived using ImPACT and the Model analysis was adopted as an estimate of the systematic uncertainties associated with the reconstruction and analysis techniques (see Sect. 5).

Additionally, the robustness of the analysis was tested using an alternative cut configuration. Within the statistical and systematic uncertainties, the results obtained with the different cut configurations were found to be in good agreement with each other.

The CT1-4 stereoscopic data collected simultaneously with the H.E.S.S. II mono data have been analysed using the H.E.S.S. I version of the Model analysis method (de Naurois \& Rolland 2009) using the loose cuts (Aharonian et al. 2006a) to ensure a low energy threshold. In total, data sets of $27.2 \mathrm{hr}$ of live time for PKS 2155-304 and 9.0 hr for PG 1553+113 have been analysed, yielding a significance of $46 \sigma$ for PKS 2155-304 and $9.0 \sigma$ for PG $1553+113$. Note that the live times differ from the corresponding mono analysis live times due to different run qualities and observation schedules for the different instruments. For each data set the spectrum is well fitted by a power-law model and the resulting forward-folded data points for PKS 2155-304 (2013 data) and PG 1553+113 are shown on Figs. 3 and 7 , respectively. The CT1-4 results for PKS 2155-304 were found to be in excellent agreement with the H.E.S.S. II mono results. Due to the limited statistics and relatively high energy threshold of the CT1-4 analysis, the CT1-4 results for PG $1553+113$ are represented on Fig. 7 by 3 data points only. Taking into consideration the systematic uncertainties on the energy scale and flux normalization (see Sect. 5), the CT1-4 data were found to be in satisfactory agreement with the CT5 results.

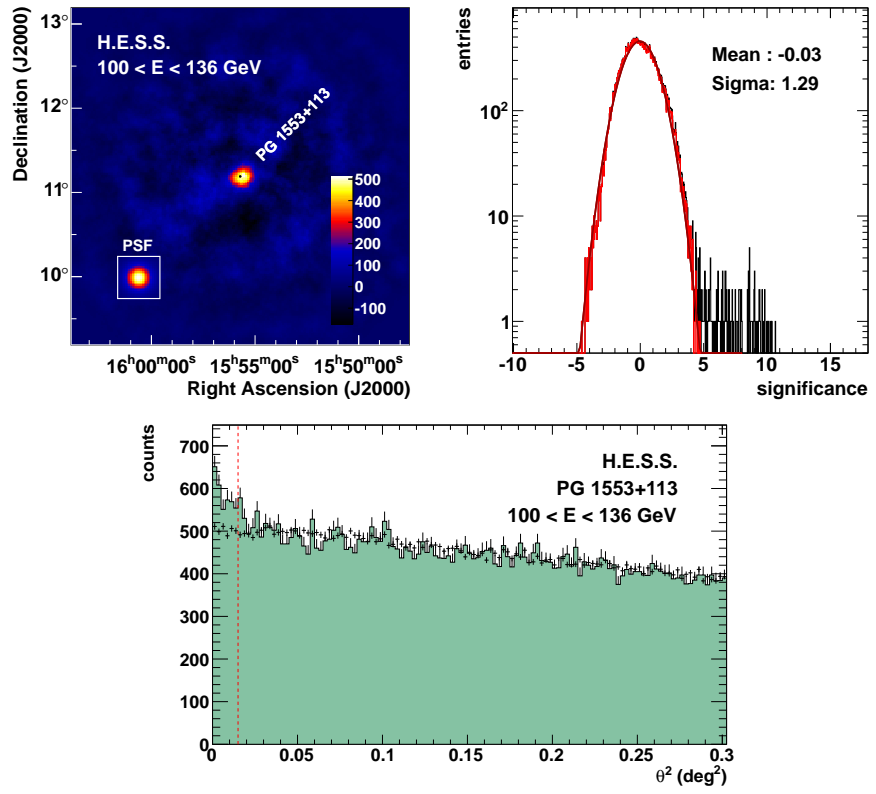

Fig. 6. Top : (Left) The PG $1553+113$ excess map and (Right) significance distribution for events with reconstructed energy between $100 \mathrm{GeV}$ and $136 \mathrm{GeV}$ (H.E.S.S. II mono analysis). Bottom: The distribution of $\theta^{2}$ (squared angular distance to PKS 2155-304) for gamma-like events.

\subsection{HE Gamma-Rays Observed by Fermi-LAT}

\subsubsection{Contemporaneous Data}

The Fermi-LAT detects gamma-ray photons above an energy of $100 \mathrm{MeV}$. Data taken contemporaneously with the H.E.S.S. II observations were analysed with the publicly available ScienceTools v10r0p5 $5^{8}$ Photon events in a circular region of $15^{\circ}$ radius centred on the position of sources of interest were considered and the PASS 8 instrument response functions (event class 128 and event type 3) corresponding to the P8R2_SOURCE_V6 response were used together with a zenith angle cut of $90^{\circ}$. The analysis was performed using the Enrico Python package (Sanchez \& Deil 2013) adapted for PASS 8 analysis. The sky model was constructed based on the 3FGL catalogue (Acero et al. 2015). The Galactic diffuse emission has been modeled using the file gll_iem_v06. fits (Acero et al. 2016) and the isotropic background using iso_P8R2_SOURCE_V6_v06.txt.

Three energy ranges were considered with the corresponding data cuts in this analysis: $0.1 \mathrm{GeV}-500 \mathrm{GeV}, 10 \mathrm{GeV}-500 \mathrm{GeV}$ and $50 \mathrm{GeV}-500 \mathrm{GeV}$, with time windows chosen to coincide with the H.E.S.S. II observation periods (as defined in Sect. 3.1).

The spectral fit parameter results are given in Table 2. For both AGNs a log-parabola fit to the contemporaneous FermiLAT data did not provide a sufficient improvement to the spectral fit, with respect to the power-law model. Some evidence for a softening of the spectrum with energy in the Fermi-LAT energy range, however, was suggested by the analysis of Fermi-LAT data for the scan of energy thresholds shown in Figs. 3 and 7 whose fit indices are given in Table 2. The data points have been obtained by redoing the Fermi-LAT analysis in a restrained energy range freezing the spectral index of the power-law model to the value found for the global fit above $100 \mathrm{MeV}$. An upper-

${ }^{8}$ See http://fermi.gsfc.nasa.gov/ssc/data/analysis/ documentation/ 
Table 2. Fermi-LAT spectral analysis results for the time intervals contemporaneous with the H.E.S.S. II observations. For each data set and energy threshold, $E_{\mathrm{th}}$, the differential flux $\phi_{0}$ at decorrelation energy $E_{0}$, photon index $\Gamma$, and value of the test statistic (TS), for the power-law fit, are provided.

\begin{tabular}{cccccccc}
\hline \hline Source & Year & MJD & $\begin{array}{c}\mathrm{E}_{\mathrm{th}} \\
(\mathrm{GeV})\end{array}$ & $\begin{array}{c}\phi_{0} \\
10^{-11}\left(\mathrm{ph} \mathrm{cm}^{-2} \mathrm{~s}^{-1} \mathrm{GeV}^{-1}\right)\end{array}$ & $\Gamma$ & $\begin{array}{c}\mathrm{E}_{0} \\
(\mathrm{GeV})\end{array}$ & TS \\
\hline PKS 2155-304 & 2013 & $56403-56601$ & 0.1 & $557 \pm 26$ & $1.82_{-0.03}^{+0.03}$ & 1.48 & 2162.6 \\
& & & 10 & $2.52 \pm 0.43$ & $2.00_{-0.21}^{+0.21}$ & 25.5 & 379.7 \\
& & 50 & $0.12 \pm 0.05$ & $1.82_{-0.72}^{+0.66}$ & 112 & 52.4 \\
PKS 2155-304 & \multirow{2}{*}{2014} & $56805-56817$ & 0.1 & $996 \pm 168$ & $1.79_{-0.13}^{+0.13}$ & 1.54 & 193.5 \\
& & & 10 & $2.36 \pm 1.18$ & $1.20_{-0.45}^{+0.45}$ & 53.3 & 52.4 \\
& & & 50 & $1.00 \pm 0.71$ & $1.53_{-1.20}^{+1.03}$ & 115 & 23.7 \\
\hline PG 1553+113 & \multirow{2}{*}{2013} & $56403-56817$ & 0.1 & $118 \pm 13$ & $1.59_{-0.07}^{+0.07}$ & 2.95 & 455.6 \\
& & & 10 & $2.04 \pm 0.53$ & $1.68_{-0.21}^{+0.26}$ & 33.5 & 169.9 \\
& & & 50 & $0.64 \pm 0.27$ & $2.97_{-1.13}^{+0.91}$ & 80.8 & 66.8 \\
\hline \hline
\end{tabular}

limit at $95 \%$ confidence level is computed if the TS is found to be below 9 .

These Fermi-LAT analysis results are used to provide gamma-ray HE-VHE SEDs of PKS 2155-304 and PG 1553+113. In Fig. 3 the 2013 H.E.S.S. II data set of PKS 2155-304 is presented along with the contemporaneous Fermi-LAT data analysed above $100 \mathrm{MeV}$ (shaded red), $10 \mathrm{GeV}$ (shaded green) and $50 \mathrm{GeV}$ (shaded magenta) respectively. These results show very good agreement between the FermiLAT and H.E.S.S. II mono data within the common overlapping region 9 , presenting a comprehensively sampled SED over more than four orders of magnitude in energy. Evidence for a strong down-turn spectral feature within this broadband SED, occurring near the transition zone between the two instruments, is apparent.

Figure 7 presents the SED of PG $1553+113$ obtained from the contemporaneous Fermi-LAT and H.E.S.S. II data. In this case, again, good agreement between the Fermi-LAT and H.E.S.S. II mono data is found within the common energy range of the two instruments. Furthermore, evidence of a strong downturn feature within this SED, occurring within the overlapping energy range of the two instruments, is once again apparent.

\subsubsection{Catalogue Data}

The H.E.S.S. II mono and contemporaneous Fermi-LAT spectra of PKS 2155-304 and PG 1553+113 obtained in the previous sections are compared here to the Fermi-LAT catalogue results. Different catalogues probing different photon statistics and energy ranges are considered here, namely the 3FGL (Acero et al. 2015), the 1FHL (Ackermann et al. 2013) and the 2FHL (Ackermann et al. 2016). The 3FGL catalogue gives an average state of the sources with 4 years of data integrated in the FermiLAT analysis above $100 \mathrm{MeV}$, while the $1 \mathrm{FHL}$ relies on the first 3 years of data with a higher energy cut at $10 \mathrm{GeV}$. Moreover, the 2FHL catalogue was built with the highest energy available to Fermi-LAT only, with $E>50 \mathrm{GeV}$, probing a somewhat different energy range, and thus potentially different spectral properties with respect to the FGL source catalogues.

The insets in Figs. 3 and 7 provide a comparison of the H.E.S.S. II mono results (shown by the blue band) with the Fermi-LAT catalogue data (red for 3FGL, green for 1FHL, and purple for 2FHL), for PKS 2155-304 and PG 1553+113, respectively.

\footnotetext{
9 80-500 GeV for PKS 2155-304 and 110-500 $\mathrm{GeV}$ for PG $1553+113$
}

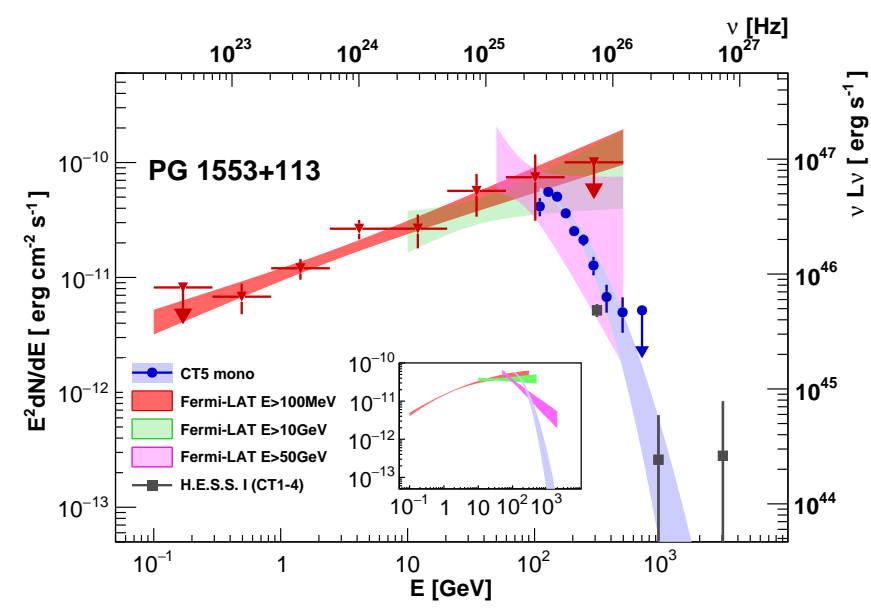

Fig. 7. The energy spectrum of PG $1553+113$ obtained from the H.E.S.S. II mono analysis (blue) in comparison with the contemporaneous Fermi-LAT data with an energy threshold of $0.1 \mathrm{GeV}$ (red triangles and confidence band), $10 \mathrm{GeV}$ (green band), and $50 \mathrm{GeV}$ (purple band) and contemporaneous CT14 data (grey squares). In all cases the bands shown represent the $1 \sigma$ confidence region. The right-hand y-axis shows the equivalent isotropic luminosity (not corrected for beaming or EBL absorption) assuming redshift $z=0.49$. The inset compares the H.E.S.S. confidence band with the Fermi-LAT catalogue data (3FGL, 1FHL and 2FHL, see Sect.4.4.2).

It is worth comparing the Fermi-LAT contemporaneous data obtained in Sect. 4.4.1 and the Fermi-LAT catalogue data discussed here. For PKS 2155-304, it is noted that the Fermi-LAT catalogue flux is slightly above the Fermi-LAT contemporaneous flux in the high energy band. For PG 1553+113, however, the catalogue flux is in close agreement with the Fermi-LAT contemporaneous flux in the high energy band. Since the FermiLAT catalogue data represent the average flux state of the source since data taking commenced in 2008, the comparable level of the fluxes (though slightly below for the case of PKS 2155-304) is suggestive that both sources were in average states of activity during the observational campaign. Note that the catalogues are based on different time intervals and different energy ranges. Furthermore, the results of the fits are dominated by the lower energy events and, in particular for the 2FHL, the statistics are rather poor at the highest energies. 


\subsection{Variability}

The AGNs considered in this work are known to be variable at VHE, both having previously been observed to exhibit major flares (Aharonian et al. 2007; Abramowski et al. 2015) In the case of PKS 2155-304, this variability has been shown to also introduce changes in the spectral shape (H.E.S.S. Collaboration et al. 2010).

In both cases, the present observational campaign found the AGNs to be in low states. For PKS 2155-304, at $E>$ $300 \mathrm{GeV}$ the spectrum level from our new H.E.S.S. II mono result agrees with the level reported for the quiescent state observed by H.E.S.S. from observations during 2005-2007 (H.E.S.S. Collaboration et al. 2010) As seen in Fig. 3, at $E<$ $300 \mathrm{GeV}$ the H.E.S.S. II mono spectrum level lies below the Fermi-LAT spectra reported in the 3FGL and 1FHL catalogues. These comparisons are all consistent with PKS 2155-304 being in a low flux state during the observations analysed in this work, as is also indicated by the Fermi-LAT contemporaneous analysis results.

Although observed in a low state, the H.E.S.S. II mono lightcurve of PKS 2155-304 did exhibit nightly and monthly variability with a fractional variability amplitude $\mathrm{F}_{\mathrm{var}}$ (Vaughan et al. 2003) of, respectively $\approx 47 \%$ and $\approx 59 \%$. Inter-year variability at VHE with a fractional variability amplitude $\mathrm{F}_{\mathrm{var}}$ of $\approx 50 \%$ has also been found. Analysis of this variability in the H.E.S.S. II mono data set revealed that an increase in the flux exists between the 2013 and the 2014 dataset by a factor $1.6 \pm 0.1_{\text {stat }}$, though without significant change in the spectral parameters. A simple power-law fit to the 2013 (resp. 2014) data yields a spectral index $\Gamma_{2013}=2.92 \pm 0.04_{\text {stat }}\left(\right.$ resp. $\Gamma_{2014}=$ $\left.2.91 \pm 0.08_{\text {stat }}\right)$. We note, however, that the statistics of the 2013 and 2014 PKS 2155-304 H.E.S.S. II mono data sets are significantly different in size. Consequently, the 2014 PKS 2155-304 data set is not sufficient to discriminate between a power-law or a log-parabola shaped spectrum, whereas the 2013 PKS 2155-304 data set is found to be significantly better fit with a log-parabolic spectrum.

For comparison, variability analysis of the PKS 2155-304 contemporaneous Fermi-LAT data, discussed in Sect.4.4.1, was carried out. Fig. 4 shows the PKS 2155-304 2013 and 2014 multi-wavelength SED obtained. It is notable that a brightening of the source flux between these two epochs by about the same level as that seen by H.E.S.S. II mono is also observed in the Fermi-LAT contemporaneous results, and again without any corresponding spectral variability. That is the Fermi-LAT and H.E.S.S. II mono photon indices are respectively consistent between the two epochs, but the overall flux increased by about $60 \%$.

The variability in HE has also been probed on a weekly timescale which gives a good balance between the ability to probe short timescale variations and good statistics. For the 2013 dataset (the 2014 dataset time range being too short), PKS 2155-304 is found to be variable with $\mathrm{F}_{\mathrm{var}}=37 \%$.

For PG 1553+113, our new H.E.S.S. II mono spectral results are in reasonable agreement with the earlier measurements by H.E.S.S. (Aharonian et al. 2008; Abramowski et al. 2015) (at $E>200 \mathrm{GeV}$ ), MAGIC (Albert et al. 2007; Aleksić et al. 2010, 2012b) and VERITAS (Aliu et al. 2015), as well as with the Fermi-LAT catalogue spectra (at $E<200 \mathrm{GeV}$ ). These comparisons with previous measurements indicate that PG 1553+113 was indeed in a low state during the H.E.S.S. II observation period of the results presented. No significant night-by-night or weekly variability is found in the H.E.S.S. II mono lightcurve.
The upper limit on $\mathrm{F}_{\mathrm{var}}$ is found to be $21 \%$ at the $95 \%$ confidence level. In the HE range, PG $1553+113$ is not variable and $\mathrm{F}_{\mathrm{var}}<110 \%$ at $95 \% \mathrm{CL}$.

\section{Systematic Uncertainties}

The main sources of systematic uncertainties in the H.E.S.S. II mono analysis presented in this publication, and their estimated contributions to the uncertainty on the spectral parameters, are summarised in Table 3 . For each source of uncertainty the table gives the flux normalisation uncertainty, the photon index uncertainty and the uncertainty on the curvature parameter $\beta$ (for the log-parabola model). In addition, the energy scale uncertainty is given in the second column. The energy scale uncertainty implies an additional uncertainty on the flux normalisation which depends on the steepness of the spectrum. It is also relevant for the determination of the position of spectral features such as the SED maximum or EBL cutoff. The procedures used here for estimating the systematic uncertainties generally repeat the procedures used for H.E.S.S. I (Aharonian et al. 2006a). We highlight that the discussion in this section focuses specifically on the sources and analysis presented. A more general discussion of the systematic uncertainties of the H.E.S.S. II mono analysis will be part of a future publication.

Except for background subtraction, all sources of uncertainty listed in Table 3 are related to the conversion of the measured event counts into flux. This conversion is done using the instrument response functions (IRF) which are determined from Monte Carlo simulations. The IRF uncertainties show how well the real instrument, after all calibrations, is described by the simulation.

The first group of uncertainties is related to the interaction of particles and their production and to the absorption of Cherenkov light in the atmosphere. The estimated uncertainty due to the shower interaction model does not exceed $1 \%$ (for photon-induced showers). The atmospheric uncertainties include the effects of the atmospheric density profile (which affects the height of shower maximum and Cherenkov light production) and the atmospheric transparency (light attenuation by Mie and Rayleigh scattering). These effects were studied extensively during H.E.S.S. phase I (Bernlöhr 2000; Aharonian et al. 2006a; Hahn et al. 2014). The uncertainties were found to be dominated by the atmospheric transparency, which has direct influence on the amount of Cherenkov light detected by the telescopes, thus affecting the energy reconstruction. Data from the telescope radiometers and other atmospheric monitoring devices, as well as trigger rate data, are used to ensure good atmospheric conditions during the observations used in the analysis (see section 3.2). For zenith angles relevant to this work, the remaining uncertainty on the absolute energy scale due to the atmosphere is estimated to be $\approx 7 \%$ (similar to the uncertainty level reported in Aharonian et al. 2006a)

The instrument simulation and calibration uncertainty includes all remaining instrumental effects, such as mirror reflectivity and electronics response. These effects are controlled using various calibration devices (Aharonian et al. 2004), as well as Cherenkov light from atmospheric muons (Leroy et al. 2003). The non-operational pixels in the CT5 camera $(<5 \%)$ and the electronics dead time $(<5 \%)$ contribute only marginally to the overall uncertainty.

The event reconstruction and selection uncertainties are derived from a comparison of the measured spectra with the results obtained using an alternative analysis chain (see Sect.4.3). 
Table 3. Estimated contributions to the systematic uncertainties in the spectral measurements using H.E.S.S. II mono for the analyses presented in this work. Numbers separated by "/" correspond to PKS 2155-304 and PG 1553+113, respectively.

\begin{tabular}{ccccc}
\hline \hline Source of Uncertainty & Energy Scale & Flux & Index & Curvature \\
\hline MC shower interactions & - & $1 \%$ & - & - \\
MC atmosphere simulation & $7 \%$ & & - & - \\
\hline Instrument simulation / calibration & $10 \%$ & $10 \%$ & - & - \\
Broken pixels & - & $5 \%$ & - & - \\
Live Time & - & $<5 \%$ & - & - \\
\hline Reconstruction and selection cuts & $15 \%$ & $15 \%$ & $0.1 / 0.46$ & $0.01 / 0.8$ \\
Background subtraction & - & $6 \% / 10 \%$ & $0.14 / 0.46$ & $0.12 / 0.6$ \\
\hline Total & $19 \%$ & $20 \% / 22 \%$ & $0.17 / 0.65$ & $0.12 / 1.0$ \\
\hline \hline
\end{tabular}

Irregularities in the camera acceptance (e.g. due to nonoperational pixels) and the night sky background (e.g. bright stars) can both have an effect on background subtraction. The background subtraction errors are controlled in this study by visually examining the raw and acceptance-corrected skymaps (to ensure that there are no artefacts, e.g. from bad calibration of individual data runs), as well as using additional dedicated tests and run quality selection. As shown already in Sect. 4, the width of the skymap significance distributions is dominated by statistical errors. This is ensured for both objects, PKS 2155-304 and PG 1553+113, and throughout the entire energy range covered by this study (see Figs. 2 and 6). Hence, arguably, the effect of the background subtraction errors should not exceed the statistical uncertainties. Consequently, the statistical uncertainties on the spectral parameters represent a reasonably conservative estimate of the background subtraction uncertainties. It should be noted, however, that the reflected-region background method, which is used for the spectral measurements, is potentially more sensitive to non-axially symmetric effects in the camera acceptance than the ring background maps (which use a 2D acceptance model). We have investigated this further by splitting the full data set into two subsets, one of which groups the data from runs taken with a wobble offset in right ascension (in either positive or negative direction) and another one for the remaining runs (with wobble in declination). The signal-to-background (S/B) ratios obtained with these subsets were compared to the full dataset $\mathrm{S} / \mathrm{B}$ ratio. It was found that the $\mathrm{S} / \mathrm{B}$ ratio varied by $\approx 3 \%$, which is about twice the background subtraction accuracy observed with the ring background method $(\approx 1.5 \%$ of the background level). Therefore in Table 3 the statistical uncertainties are doubled to obtain the values for the background subtraction uncertainties.

The net effect of all uncertainties summed in quadrature is given in the last row of Table 3. It can be noted that the spectral index and curvature uncertainties are dominated by the reconstruction, event selection and background subtraction uncertainties, while the description of the atmosphere and instrument calibration contribute substantially to the energy scale and flux normalisation uncertainties.

It should lastly be highlighted that the systematic uncertainties are energy-dependent. In particular, the background subtraction uncertainties tend to become more important towards low energies, where the signal-to-background ratio is usually smaller. For an analysis aiming at the lowest energies this can lead to a large uncertainty in the measurement of spectral index and curvature, especially for soft spectrum sources, as is the case for PG $1553+113$.

In the context of variability studies, the uncertainty values presented in Table 3 can be considered as a conservative upper bound. Preliminary studies of steady sources with H.E.S.S. II suggest that the RMS variability induced by systematic effects is about $15-20 \%$, a result similar to that for H.E.S.S. I (Aharonian et al. 2006a). This suggests that at least some of the spectral measurement uncertainties are constant in time and could therefore be reduced by means of additional calibrations. Variations related to changes in the atmosphere transparency can also be reduced by means of additional corrections (Hahn et al. 2014).

\section{Discussion}

The successful H.E.S.S. II mono observations and analysis of PKS 2155-304 and PG 1553+113 convincingly demonstrate that the low energy part of the VHE spectrum is accessible to the H.E.S.S. experiment, following the addition of the CT5 instrument. This fact makes EBL studies of high redshift AGNs by H.E.S.S. II mono feasible, without the need for strong theoretical biases on the intrinsic spectra or the need to rely on spectral extrapolations using results from other instruments.

Here we consider EBL deabsorbed fits to the H.E.S.S. II mono and contemporaneous Fermi-LAT spectra for both AGNs. Our aim here is twofold. The first is to investigate evidence for curvature in the two AGN intrinsic spectra, correcting for EBL absorption effects. Second, given the present systematic uncertainties derived for these data sets, we determine the corresponding uncertainties on the combined fit parameters. Such considerations provide insight into the constraining power of these results, under the assumption of both a specific EBL model (in this work the one of Franceschini et al. 2008) and simple underlying spectral shape.

The spectra in the H.E.S.S. II mono energy range have been reconstructed with a spectral model corrected for EBL absorption. Furthermore, for PG $1553+113$, whose redshift is not wellconstrained, we adopt the well-motivated value of $z=0.49$ (Abramowski et al. 2015).

In order to look for a possible turnover in the intrinsic spectrum and, if present, to locate the peak emission in the energy flux $\left(E^{2} \mathrm{~d} N / \mathrm{d} E\right)$ representation, the EBL deabsorbed Fermi-LAT and H.E.S.S. II mono data points were fitted both separately and as a combined data set with power-law, broken power-law and log-parabola models. In the combined fit procedure, a consideration of the systematic uncertainties for each of the data sets was taken into account in the analysis.

For the H.E.S.S. systematic uncertainties, the effect of the energy systematic uncertainty on the deabsorbed spectrum fit results was found to be the dominant contributing systematic. The contribution of this uncertainty on the results was estimated through the shifting of the data points in the $E d N / d E$ representation by an energy scale factor of $19 \%$ (see Table 3 ) before applying the EBL deabsorbtion. The variation in the best-fit model, introduced via the application of this procedure within 
Table 4. Parameters obtained for the combined fit of the Fermi-LAT and H.E.S.S. data. The reference energy $E_{0}$ used here is 100 $\mathrm{GeV}$. For both blazars, the log-parabola fits values are provided. For PG 1553+113, the values for the power-law model, which was marginally disfavoured, are also given. The last column gives the significance, obtained by comparing the $\chi^{2}$ values for the log-parabola model against those for the power-law model, using only statistical errors in the analysis.

\begin{tabular}{|c|c|c|c|c|c|}
\hline Source & $\phi_{0}\left[10^{-11} \mathrm{~cm}^{-2} \mathrm{~s}^{-1}\right]$ & $\Gamma$ & $\beta$ & $\log _{10}\left(E_{\text {peak }}[\mathrm{GeV}]\right)$ & Sig. $(\sigma)$ \\
\hline PKS 2155-304 & $2.35 \pm 0.10_{\text {stat }} \pm 0.57_{\text {sys }}$ & $2.30 \pm 0.04_{\text {stat }} \pm 0.09_{\text {sys }}$ & $0.15 \pm 0.02_{\text {stat }} \pm 0.02_{\text {sys }}$ & $0.99 \pm 0.19_{\text {stat }} \pm 0.19_{\text {sys }}$ & 5.1 \\
\hline PG 1553+113 & $5.97 \pm 0.25_{\text {stat }} \pm 2.19_{\text {sys }}$ & $1.68 \pm 0.05_{\text {stat }} \pm 0.13_{\text {sys }}$ & - & - & - \\
\hline PG $1553+113$ & $6.66 \pm 0.42_{\text {stat }} \pm 1.43_{\text {sys }}$ & $1.83 \pm 0.08_{\text {stat }} \pm 0.29_{\text {sys }}$ & $0.12 \pm 0.05_{\text {stat }} \pm 0.13_{\text {sys }}$ & $2.76 \pm 0.45_{\text {stat }} \pm 0.93_{\text {sys }}$ & 2.2 \\
\hline
\end{tabular}

the full energy uncertainty range, was then taken as the systematic contribution to the uncertainty on each model parameter (see Table 4). An estimate of the size of the Fermi-LAT systematic uncertainties was also obtained, using the effective area systematic uncertainty, derived by the LAT collaboration ${ }^{10}$ These uncertainties were noted to be small in comparison to the statistical errors such that their further consideration could be safely neglected.

In the case of PKS 2155-304, separate fits of the FermiLAT and H.E.S.S. II mono EBL deabsorbed data, the power-law model was found to provide a sufficient description in both cases. The power-law fit of the H.E.S.S. II mono 2013 data obtained an intrinsic spectral index of $\Gamma=2.49 \pm 0.05$. Such an index appears somewhat softer than the power-law analysis of the Fermi-LAT contemporaneous data $(\Gamma=1.82 \pm 0.03$ see Table 2). The spectral fits found for the combined data sets, dominated by the low energy data points where EBL effects can be neglected, allowed the continuity of the source spectrum to be probed. The fit of the combined Fermi-LAT and H.E.S.S. II mono data with a logparabola model was preferred at the $5.1 \sigma$ level with respect to the power-law model (See Figure 8). The broken power-law does not significantly improve the fit in this case. The results of the fit are given in Table 4. The peak flux position within the SED was at a moderate energy (around $10 \mathrm{GeV}$ ), in agreement with its 4-year averaged position found in the 3FGL.

For PG $1553+113$, an EBL absorbed power-law fit to the H.E.S.S. II mono spectra required an intrinsic spectral index of $\Gamma=1.91 \pm 0.13$. For comparison, Table 2 shows that the FermiLAT spectral fits for power laws with thresholds of $100 \mathrm{MeV}$ and $10 \mathrm{GeV}$ give consistent spectral indices to this value. On the other hand, the fit of the combined Fermi-LAT and H.E.S.S. II mono gamma-ray data, however, found a log-parabola model preferred at the $2.2 \sigma$ level over the power-law model (See Figure 9). The fit values for these two spectral models are provided in Table 4 . The parameters that results from fits with a broken power-law being close to one of the single power-law model case. The sizeable systematic errors, once also taken into account, however, weaken this preference. Thus, this only marginal improvement, brought by the log-parabola model, suggests that the observed softening of the PG $1553+113$ spectrum is predominantly introduced by VHE interaction on the EBL, a result consistent with that from other instruments which have searched for intrinsic curvature in the source's spectra (Aleksić et al. 2015b) Furthermore, the constraint on the intrinsic peak position, at a value of $0.6_{-0.4}^{+1.0} \mathrm{TeV}$, also carries significant uncertainties. This limitation is primarily due to the relatively small intrinsic curvature, limited lever arm (energy range coverage by the measurements), and the very soft observed spectral index in the H.E.S.S. II mono band, which amplifies the effect of the energy scale uncertainty. This could be improved in the future via more

10 see http://fermi.gsfc.nasa.gov/ssc/data/analysis/ scitools/Aeff_Systematics.html

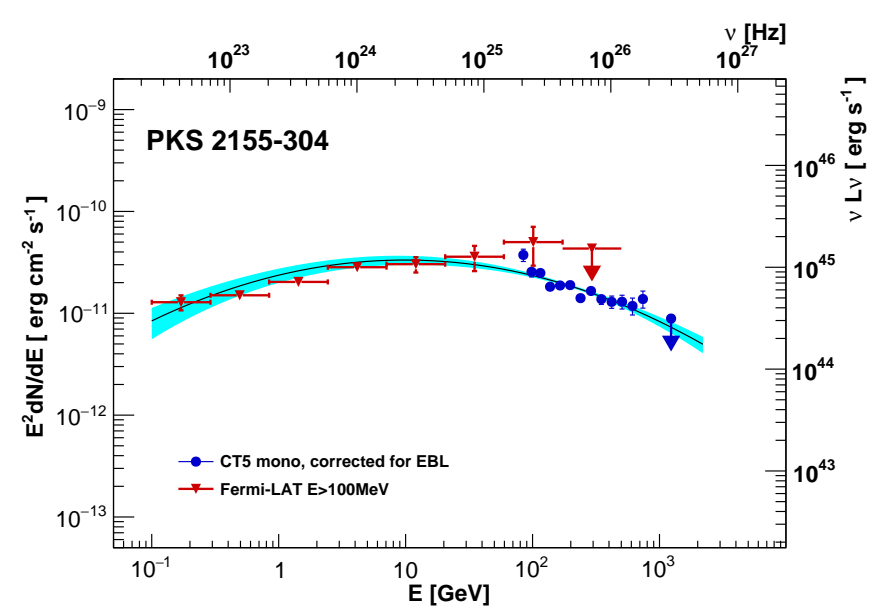

Fig. 8. The energy spectrum of PKS 2155-304 obtained from the H.E.S.S. II mono analysis (blue) of the 2013 data corrected for EBL absorption in comparison with the contemporaneous Fermi-LAT data with a minimal energy of $0.1 \mathrm{GeV}$ (red). The black line is the best-fit log-parabola model to the points and the cyan butterfly indicates the $1 \sigma$ region using only the statistical errors in the combined data set analysis. The right-hand $\mathrm{y}$-axis shows the equivalent isotropic luminosity (not beaming corrected).

accurate calibration of the H.E.S.S. II mono energy scale, using bright flaring or stable sources to compare flux measurements with those of Fermi-LAT contemporaneous measurements as e.g in Meyer et al. (2010).

In summary, the high-energy SED of PG $1553+113$, corrected for EBL with the model of Franceschini et al. (2008), assuming a redshift of 0.49 , reveals only marginal evidence for intrinsic curvature once systematic uncertainties are taken into account. This result is compatible with a scenario in which the observed spectral downturn at an energy of around $100 \mathrm{GeV}$ is introduced through the attenuation at the highest energies is due to the interaction of VHE photons with the EBL. Contrary to this, in the case of PKS 2155-304, the EBL corrected SED is better described by a log-parabola model than by a power-law. The addition of intrinsic spectral curvature or break is required to account for the data presented. Such a feature is naturally expected rather generically on physical grounds in the high energy region of the particle spectrum for both stochastic and shock acceleration mechanisms (Park \& Petrosian 1995; Heavens \& Meisenheimer 1987; Kirk et al. 1998)

\section{Conclusions}

Here we report, for the first time, H.E.S.S. II mono blazar results following observations of PKS 2155-304 in 2013 and 2014 and PG $1553+113$ in 2013, taken with the new CT5 instrument in 


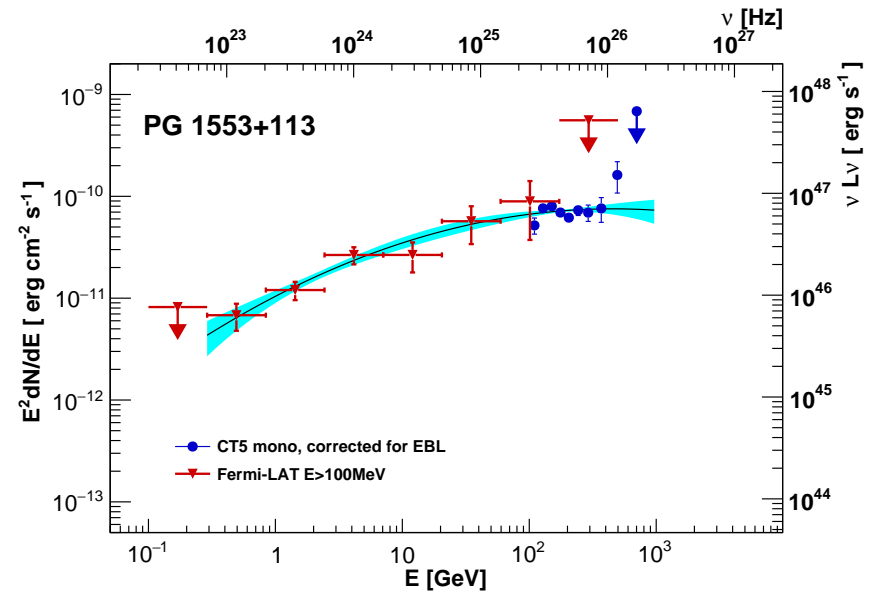

Fig. 9. The energy spectrum of PG $1553+113$ obtained from the H.E.S.S. II mono analysis (blue) corrected for EBL absorption in comparison with the contemporaneous Fermi-LAT data with a minimal energy of $0.1 \mathrm{GeV}$ (red). The assumed redshift is $\mathrm{z}=0.49$. The black line is the best-fit log-parabola model fit to the points and the cyan butterfly indicates the $1 \sigma$ (statistical error only) uncertainty region. The right-hand y-axis shows the equivalent isotropic luminosity (not beaming corrected).

monoscopic configuration. The successful analysis of these observations resulted in the detection of these two AGNs at levels of $\sim 42 \sigma(36 \sigma)$ and $\sim 27 \sigma(21 \sigma)$, respectively. For these results, low-energy thresholds of $80 \mathrm{GeV}$ and $110 \mathrm{GeV}$, respectively, were achieved. These thresholds amount to a reduction by a factor of 2-3 relative to that achieved in the CT1-4 crosscheck results presented (see Figures 3 and 7). Furthermore, we note that the energy threshold achieved by the present H.E.S.S. II mono analysis remains limited by the accuracy of the background subtraction method, rather than by the instrument trigger threshold.

Namely, at energies below the respective thresholds achieved for the PKS 2155-304 and PG 1553+113 datasets, the systematic uncertainties in background subtraction become larger than the statistical uncertainties. The energy at which the transition from statistics-dominated to systematics-dominated regime occurs depends on the accuracy of background subtraction and the size of the dataset being analysed. For the present analysis the level of systematic uncertainties in background subtraction was found to be $\approx 1.5 \%$ (for skymaps), which corresponds to a minimal requirement for the signal-to-background ratio of $S / B>7.5 \%$ for a $5 \sigma$ detection (assuming normally distributed errors). This limitation does not apply to the special case of gamma-ray pulsars, where the pulsar phasogram can be used to define "off regions" for background subtraction. Subsequent improvements and reduction in the energy threshold are likely to occur in the future.

A comparison of the emission level of PKS 2155-304 and PG $1553+113$ with their historic observations revealed both to be in low states of activity, with PKS 2155-304 found to be within $1 \sigma$ of its mean quiescent level, as defined in H.E.S.S. Collaboration et al. (2010), during the 2013 H.E.S.S. II observations. Temporal analysis of its emission during the campaign revealed mild $(\sim 50 \%)$ variability in the lightcurve of PKS 2155-304 between the 2013 and 2014 H.E.S.S. II data sets. No significant variability was found in the emission of PG 1553+113. Further analysis of the PKS 2155-304 data, separating the two consecutive years of observations, revealed an enhancement in the flux state, by a factor of $\sim 60 \%$, in the 2014 data. Interestingly, a similar size increase in the flux level between the 2013 and 2014 fluxes is seen in the contemporaneous Fermi-LAT data (see Fig. 4). Spectral analysis of the fluxes from these two different brightness periods, however, reveals no evidence for significant alteration of the spectral shape from either the H.E.S.S. II mono or Fermi-LAT observations. The change in source state between these periods therefore appears to be associated with a broad increase in the source brightness in the $0.1-1000 \mathrm{GeV}$ energy range.

Multi-wavelength SED plots containing the new H.E.S.S. II data points for these observations of PKS 2155-304 and PG 1553+113, and their comparison with contemporaneous Fermi-LAT observations, are shown in Figs. 3, 4, and 7. Spectral analysis of the H.E.S.S. II mono data indicate that a log-parabola fit is preferred over a simple power-law or a broken power-law fit in both cases. The measurement of the curvature parameter in these fits, however, is marginal for PG $1553+113$ once the systematic errors are taken into account. Within their multiwavelength SEDs, the presence of a strong spectral downturn feature, at an energy of $\sim 100 \mathrm{GeV}$, is apparent in both cases, consistent with previous multi-wavelength observations made of these objects during low activity states (Aharonian et al. 2009; H.E.S.S. Collaboration et al. 2014b; Abdo et al. 2010; Aleksić et al. 2012b; Aliu et al. 2015). The introduction of such a feature at these energies is expected through gamma-ray absorption on the EBL during their transit through extragalactic space. Adopting a specific EBL model, spectral fitting of the data, deabsorbed on the EBL, indicates the presence of significant curvature in the intrinsic source spectrum for PKS 2155-304, with the peak of the intrinsic SED sitting at an energy of $\sim 10 \mathrm{GeV}$. A similar EBL deabsorbed analysis for PG $1553+113$ reveals a milder level of curvature in the intrinsic spectrum, suggesting that the peak of the intrinsic SED sits at an energy of $\sim 500 \mathrm{GeV}$. However, once systematic errors are taken into account, the intrinsic spectrum of PG $1553+113$ was found to be consistent with no curvature. It therefore remains possible that the observed softening in the PG $1553+113$ spectra is purely introduced by VHE interaction on the EBL, and is not intrinsic to the source.

Our results demonstrate for the first time the successful employment of the monoscopic data from the new H.E.S.S. II instrument (CT5) for blazar and other AGN studies. These results mark a significant step forward in lowering the gamma-ray energy range that AGN may be probed in the H.E.S.S. II era. This reduction in the energy threshold opens up the opportunity to probe new low-energy aspects about AGN fluxes, their variability, and their attenuation on the EBL out to larger redshifts than that probed previously in the H.E.S.S. I era. Furthermore, coupled with the level of significance obtained for the detection of both AGNs, the reduction in threshold offers great potential for temporally resolving AGN lightcurves down to unprecedented temporal scales during flaring episodes.

Acknowledgements. The support of the Namibian authorities and of the University of Namibia in facilitating the construction and operation of H.E.S.S. is gratefully acknowledged, as is the support by the German Ministry for Education and Research (BMBF), the Max Planck Society, the German Research Foundation (DFG), the French Ministry for Research, the CNRS-IN2P3 and the Astroparticle Interdisciplinary Programme of the CNRS, the U.K. Science and Technology Facilities Council (STFC), the IPNP of the Charles University, the Czech Science Foundation, the Polish Ministry of Science and Higher Education, the South African Department of Science and Technology and National Research Foundation, the University of Namibia, the Innsbruck University, the Austrian Science Fund (FWF), and the Austrian Federal Ministry for Science, Research and Economy, and by the University of Adelaide and the Australian Research Council. We appreciate the excellent work of the technical support staff in Berlin, Durham, Hamburg, Heidelberg, Palaiseau, Paris, Saclay, and in Namibia in the 
construction and operation of the equipment. This work benefited from services provided by the H.E.S.S. Virtual Organisation, supported by the national resource providers of the EGI Federation.

The Fermi-LAT Collaboration acknowledges generous ongoing support from a number of agencies and institutes that have supported both the development and the operation of the LAT as well as scientific data analysis. These include the National Aeronautics and Space Administration and the Department of Energy in the United States, the Commissariat à l'Energie Atomique and the Centre National de la Recherche Scientifique / Institu National de Physique Nucléaire et de Physique des Particules in France, the Agenzia Spaziale Italiana and the Istituto Nazionale di Fisica Nucleare in Italy, the Ministry of Education, Culture, Sports, Science and Technology (MEXT), High Energy Accelerator Research Organization (KEK) and Japan Aerospace Exploration Agency (JAXA) in Japan, and the K. A. Wallenberg Foundation, the Swedish Research Council and the Swedish National Space Board in Sweden.

Additional support for science analysis during the operations phase is gratefully acknowledged from the Istituto Nazionale di Astrofisica in Italy and the Centre National d'Études Spatiales in France.

This research has made use of NASA's Astrophysics Data System. This research has made use of the SIMBAD database, operated at CDS, Strasbourg, France. This research made use of Enrico, a community-developed Python package to simplify Fermi-LAT analysis (Sanchez \& Deil 2013)

\section{References}

Abdo, A. A., Ackermann, M., Ajello, M., et al. 2009, ApJS, 183, 46 Abdo, A. A., Ackermann, M., Ajello, M., et al. 2010, ApJ, 708, 1310 Abeysekara, A. U., Archambault, S., Archer, A., et al. 2015, ApJ, 815, L22 Abramowski, A., Aharonian, F., Ait Benkhali, F., et al. 2015, ApJ, 802, 65

Acero, F., Ackermann, M., Ajello, M., et al. 2015, ApJS, 218, 23

Acero, F., Ackermann, M., Ajello, M., et al. 2016, ApJS, 223, 26

Ackermann, M., Ajello, M., Albert, A., et al. 2015, ApJ, 813, L41

Ackermann, M., Ajello, M., Allafort, A., et al. 2013, ApJS, 209, 34

Ackermann, M., Ajello, M., Atwood, W. B., et al. 2016, ApJS, 222, 5

Aharonian, F., Akhperjanian, A. G., Anton, G., et al. 2009, ApJ, 696, L150

Aharonian, F., Akhperjanian, A. G., Aye, K.-M., et al. 2005, A\&A, 430, 865

Aharonian, F., Akhperjanian, A. G., Aye, K.-M., et al. 2004, Astroparticle Physics, 22, 109

Aharonian, F., Akhperjanian, A. G., Barres de Almeida, U., et al. 2008, A\&A 477,481

Aharonian, F., Akhperjanian, A. G., Bazer-Bachi, A. R., et al. 2007, ApJ, 664, L71

Aharonian, F., Akhperjanian, A. G., Bazer-Bachi, A. R., et al. 2006a, A\&A, 457, 899

Aharonian, F., Akhperjanian, A. G., Bazer-Bachi, A. R., et al. 2006b, A\&A, 448, L19

Albert, J., Aliu, E., Anderhub, H., et al. 2007, ApJ, 654, L119

Aleksić, J., Alvarez, E. A., Antonelli, L. A., et al. 2012a, A\&A, 544, A75

Aleksić, J., Alvarez, E. A., Antonelli, L. A., et al. 2012b, ApJ, 748, 46

Aleksić, J., Anderhub, H., Antonelli, L. A., et al. 2010, A\&A, 515, A76

Aleksić, J., Ansoldi, S., Antonelli, L. A., et al. 2015a, Journal of High Energy Astrophysics, 5, 30

Aleksić, J., Ansoldi, S., Antonelli, L. A., et al. 2015b, MNRAS, 450, 4399

Aliu, E., Archer, A., Aune, T., et al. 2015, ApJ, 799, 7

Berge, D., Funk, S., \& Hinton, J. 2007, A\&A, 466, 1219

Bernlöhr, K. 2000, Astroparticle Physics, 12, 255

Biteau, J. \& Williams, D. A. 2015, ApJ, 812, 60

Bolmont, J., Corona, P., Gauron, P., et al. 2014, Nuclear Instruments and Methods in Physics Research A, 761, 46

Chadwick, P. M., Lyons, K., McComb, T. J. L., et al. 1999, ApJ, 513, 161

Danforth, C. W., Keeney, B. A., Stocke, J. T., Shull, J. M., \& Yao, Y. 2010, ApJ, 720,976

de Naurois, M. \& Rolland, L. 2009, Astroparticle Physics, 32, 231

Falomo, R., Pesce, J. E., \& Treves, A. 1993, ApJ, 411, L63

Franceschini, A., Rodighiero, G., \& Vaccari, M. 2008, A\&A, 487, 837

Ganguly, R., Lynch, R. S., Charlton, J. C., et al. 2013, MNRAS, 435, 1233

Griffiths, R. E., Briel, U., Chaisson, L., \& Tapia, S. 1979, ApJ, 234, 810

Hahn, J., de los Reyes, R., Bernlöhr, K., et al. 2014, Astroparticle Physics, 54, 25

Heavens, A. F. \& Meisenheimer, K. 1987, MNRAS, 225, 335

H.E.S.S. Collaboration, Abramowski, A., Acero, F., et al. 2010, A\&A, 520, A83

H.E.S.S. Collaboration, Abramowski, A., Aharonian, F., et al. 2014a, A\&A, 564, A9

H.E.S.S. Collaboration, Abramowski, A., Aharonian, F., et al. 2014b, A\&A, 571, A39

Holler, M., Balzer, A., Chalmé-Calvet, R., et al. 2015, in Proceedings of the 34th International Cosmic Ray Conference (ICRC 2015)
Kirk, J. G., Rieger, F. M., \& Mastichiadis, A. 1998, A\&A, 333, 452

Kotilainen, J. K., Falomo, R., \& Scarpa, R. 1998, A\&A, 336, 479

Leroy, N., Bolz, O., Guy, J., et al. 2003, International Cosmic Ray Conference, 5,2895

Li, T.-P. \& Ma, Y.-Q. 1983, ApJ, 272, 317

Mazin, D. \& Goebel, F. 2007, ApJ, 655, L13

Meyer, M., Horns, D., \& Zechlin, H.-S. 2010, A\&A, 523, A2

Park, B. T. \& Petrosian, V. 1995, ApJ, 446, 699

Parsons, R. D., Gajdus, M., Murach, T., \& for the H. E. S. S. collaboration. 2015, in Proceedings of the 34th International Cosmic Ray Conference (ICRC 2015)

Parsons, R. D. \& Hinton, J. A. 2014, Astroparticle Physics, 56, 26

Piron, F., Djannati-Atai, A., Punch, M., et al. 2001, A\&A, 374, 895

Sanchez, D. A. \& Deil, C. 2013, in Proceedings of the 33rd International Cosmic Ray Conference (ICRC 2013)

Schwartz, D. A., Griffiths, R. E., Schwarz, J., Doxsey, R. E., \& Johnston, M. D. 1979, ApJ, 229, L53

Vaughan, S., Edelson, R., Warwick, R. S., \& Uttley, P. 2003, MNRAS, 345, 1271

Vestrand, W. T., Stacy, J. G., \& Sreekumar, P. 1995, IAU Circ., 6169

1 Centre for Space Research, North-West University, Potchefstroom 2520, South Africa

2 Universität Hamburg, Institut für Experimentalphysik, Luruper Chaussee 149, D 22761 Hamburg, Germany

3 Max-Planck-Institut für Kernphysik, P.O. Box 103980, D 69029 Heidelberg, Germany

4 Dublin Institute for Advanced Studies, 31 Fitzwilliam Place, Dublin 2, Ireland

5 National Academy of Sciences of the Republic of Armenia, Marshall Baghramian Avenue, 24, 0019 Yerevan, Republic of Armenia

6 Yerevan Physics Institute, 2 Alikhanian Brothers St., 375036 Yerevan, Armenia

7 Institut für Physik, Humboldt-Universität zu Berlin, Newtonstr. 15, D 12489 Berlin, Germany

8 University of Namibia, Department of Physics, Private Bag 13301, Windhoek, Namibia

9 GRAPPA, Anton Pannekoek Institute for Astronomy, University of Amsterdam, Science Park 904, 1098 XH Amsterdam, The Netherlands

10 Department of Physics and Electrical Engineering, Linnaeus University, 35195 Växjö, Sweden

11 Institut für Theoretische Physik, Lehrstuhl IV: Weltraum und Astrophysik, Ruhr-Universität Bochum, D 44780 Bochum, Germany

12 GRAPPA, Anton Pannekoek Institute for Astronomy and Institute of High-Energy Physics, University of Amsterdam, Science Park 904, 1098 XH Amsterdam, The Netherlands

13 Institut für Astro- und Teilchenphysik, Leopold-FranzensUniversität Innsbruck, A-6020 Innsbruck, Austria

14 School of Physical Sciences, University of Adelaide, Adelaide 5005, Australia

15 LUTH, Observatoire de Paris, PSL Research University, CNRS, Université Paris Diderot, 5 Place Jules Janssen, 92190 Meudon, France

16 Sorbonne Universités, UPMC Université Paris 06, Université Paris Diderot, Sorbonne Paris Cité, CNRS, Laboratoire de Physique 
Nucléaire et de Hautes Energies (LPNHE), 4 place Jussieu, F75252, Paris Cedex 5, France

17 DSM/Irfu, CEA Saclay, F-91191 Gif-Sur-Yvette Cedex, France

18 Astronomical Observatory, The University of Warsaw, Al. Ujazdowskie 4, 00-478 Warsaw, Poland

19 Aix Marseille Université, CNRS/IN2P3, CPPM UMR 7346, 13288 Marseille, France

20 Instytut Fizyki Jạdrowej PAN, ul. Radzikowskiego 152, 31-342 Kraków, Poland

21 Funded by EU FP7 Marie Curie, grant agreement No. PIEF-GA2012-332350,

22 Laboratoire Univers et Particules de Montpellier, Université Montpellier, CNRS/IN2P3, CC 72, Place Eugène Bataillon, F34095 Montpellier Cedex 5, France

23 School of Physics, University of the Witwatersrand, 1 Jan Smuts Avenue, Braamfontein, Johannesburg, 2050 South Africa

${ }^{24}$ Laboratoire d'Annecy-le-Vieux de Physique des Particules, Université Savoie Mont-Blanc, CNRS/IN2P3, F-74941 Annecy-leVieux, France

25 Landessternwarte, Universität Heidelberg, Königstuhl, D 69117 Heidelberg, Germany

${ }^{26}$ Université Bordeaux, CNRS/IN2P3, Centre d'Études Nucléaires de Bordeaux Gradignan, 33175 Gradignan, France

27 Oskar Klein Centre, Department of Physics, Stockholm University, Albanova University Center, SE-10691 Stockholm, Sweden

28 Wallenberg Academy Fellow,

29 Institut für Astronomie und Astrophysik, Universität Tübingen, Sand 1, D 72076 Tübingen, Germany

30 Laboratoire Leprince-Ringuet, Ecole Polytechnique, CNRS/IN2P3, F-91128 Palaiseau, France

31 APC, AstroParticule et Cosmologie, Université Paris Diderot, CNRS/IN2P3, CEA/Irfu, Observatoire de Paris, Sorbonne Paris Cité, 10, rue Alice Domon et Léonie Duquet, 75205 Paris Cedex 13, France

32 Univ. Grenoble Alpes, IPAG, F-38000 Grenoble, France CNRS, IPAG, F-38000 Grenoble, France

33 Department of Physics and Astronomy, The University of Leicester, University Road, Leicester, LE1 7RH, United Kingdom

${ }^{34}$ Nicolaus Copernicus Astronomical Center, ul. Bartycka 18, 00-716 Warsaw, Poland

35 Institut für Physik und Astronomie, Universität Potsdam, KarlLiebknecht-Strasse 24/25, D 14476 Potsdam, Germany

36 Friedrich-Alexander-Universität Erlangen-Nürnberg, Erlangen Centre for Astroparticle Physics, Erwin-Rommel-Str. 1, D 91058 Erlangen, Germany

37 DESY, D-15738 Zeuthen, Germany

38 Obserwatorium Astronomiczne, Uniwersytet Jagielloński, ul. Orla 171, 30-244 Kraków, Poland

39 Centre for Astronomy, Faculty of Physics, Astronomy and Informatics, Nicolaus Copernicus University, Grudziadzka 5, 87100 Torun, Poland

40 Department of Physics, University of the Free State, PO Box 339, Bloemfontein 9300, South Africa

${ }^{41}$ Heisenberg Fellow (DFG), ITA Universität Heidelberg, Germany

42 GRAPPA, Institute of High-Energy Physics, University of Amsterdam, Science Park 904, 1098 XH Amsterdam, The Netherlands

43 Department of Physics, Rikkyo University, 3-34-1 Nishi-Ikebukuro, Toshima-ku, Tokyo 171-8501, Japan

44 Now at Santa Cruz Institute for Particle Physics and Department of Physics, University of California at Santa Cruz, Santa Cruz, CA 95064, USA

45 Deutsches Elektronen Synchrotron DESY, D-15738 Zeuthen, Germany

46 Department of Physics and Astronomy, Clemson University, Kinard Lab of Physics, Clemson, SC 29634-0978, USA

47 Università di Pisa and Istituto Nazionale di Fisica Nucleare, Sezione di Pisa I-56127 Pisa, Italy

48 W. W. Hansen Experimental Physics Laboratory, Kavli Institute for Particle Astrophysics and Cosmology, Department of Physics and SLAC National Accelerator Laboratory, Stanford University, Stanford, CA 94305, USA

49 Istituto Nazionale di Fisica Nucleare, Sezione di Trieste, I-34127 Trieste, Italy

50 Dipartimento di Fisica, Università di Trieste, I-34127 Trieste, Italy

51 Istituto Nazionale di Fisica Nucleare, Sezione di Pisa, I-56127 Pisa, Italy

52 Istituto Nazionale di Fisica Nucleare, Sezione di Torino, I-10125 Torino, Italy

53 Dipartimento di Fisica, Università degli Studi di Torino, I-10125 Torino, Italy

${ }^{54}$ Laboratoire Univers et Particules de Montpellier, Université Montpellier, CNRS/IN2P3, F-34095 Montpellier, France

55 Consorzio Interuniversitario per la Fisica Spaziale (CIFS), I-10133 Torino, Italy

56 Dipartimento di Fisica “M. Merlin” dell’Università e del Politecnico di Bari, I-70126 Bari, Italy

57 Istituto Nazionale di Fisica Nucleare, Sezione di Bari, I-70126 Bari, Italy

58 INAF-Istituto di Astrofisica Spaziale e Fisica Cosmica, I-20133 Milano, Italy

59 Agenzia Spaziale Italiana (ASI) Science Data Center, I-00133 Roma, Italy

${ }^{60}$ Istituto Nazionale di Fisica Nucleare, Sezione di Perugia, I-06123 Perugia, Italy

61 Dipartimento di Fisica, Università degli Studi di Perugia, I-06123 Perugia, Italy

62 Dipartimento di Fisica e Astronomia "G. Galilei", Università di Padova, I-35131 Padova, Italy

63 INAF Istituto di Radioastronomia, I-40129 Bologna, Italy

${ }^{64}$ Dipartimento di Astronomia, Università di Bologna, I-40127 Bologna, Italy

65 Università Telematica Pegaso, Piazza Trieste e Trento, 48, I-80132 Napoli, Italy

${ }^{66}$ Università di Udine, I-33100 Udine, Italy

67 Istituto Nazionale di Fisica Nucleare, Sezione di Padova, I-35131 Padova, Italy

${ }^{68}$ Laboratoire de Physique et Chimie de l'Environnement et de l'Espace - Université d'Orléans / CNRS, F-45071 Orléans Cedex 02, France

69 Station de radioastronomie de Nançay, Observatoire de Paris, CNRS/INSU, F-18330 Nançay, France

70 NASA Goddard Space Flight Center, Greenbelt, MD 20771, USA

71 NASA Postdoctoral Program Fellow, USA

72 Science Institute, University of Iceland, IS-107 Reykjavik, Iceland

73 Department of Physics, Graduate School of Science, University of Tokyo, 7-3-1 Hongo, Bunkyo-ku, Tokyo 113-0033, Japan

74 Department of Physical Sciences, Hiroshima University, HigashiHiroshima, Hiroshima 739-8526, Japan

75 Department of Physics, KTH Royal Institute of Technology, AlbaNova, SE-106 91 Stockholm, Sweden

76 The Oskar Klein Centre for Cosmoparticle Physics, AlbaNova, SE10691 Stockholm, Sweden

77 Institute of Space Sciences (IEEC-CSIC), Campus UAB, E-08193 Barcelona, Spain

78 Space Science Division, Naval Research Laboratory, Washington, DC 20375-5352, USA

79 Hiroshima Astrophysical Science Center, Hiroshima University, Higashi-Hiroshima, Hiroshima 739-8526, Japan

80 Istituto Nazionale di Fisica Nucleare, Sezione di Roma "Tor Vergata”, I-00133 Roma, Italy

81 Max-Planck-Institut für Physik, 80805 München, Germany

82 Erlangen Centre for Astroparticle Physics, D-91058 Erlangen, Germany

83 Funded by contract FIRB-2012-RBFR12PM1F from the Italian Ministry of Education, University and Research (MIUR)

84 NYCB Real-Time Computing Inc., Lattingtown, NY 11560-1025, USA

85 Institució Catalana de Recerca i Estudis Avançats (ICREA), Barcelona, Spain

86 INAF-IASF Bologna, I-40129 Bologna, Italy

87 Department of Physics and Department of Astronomy, University of Maryland, College Park, MD 20742, USA

88 Department of Physics, Rikkyo University, 3-34-1 Nishi-Ikebukuro, Toshima-ku, Tokyo 171-8501, Japan 